\title{
Strongly Regular Graphs and Partial Geometries
}

\author{
A.E. Brouwer \\ J.H. van Lint
}

\begin{abstract}
We give a survey of recent results concerning construction, uniqueness or nonexistence of strongly regular graphs and partial geometries. Essentially we restrict ourselves to results which did not occur in the well known earlier surveys.
\end{abstract}

\section{Introduction}

Since the introduction of strongly regular graphs and partial geometries by Bose [4] several surveys of the theory and of constructions have appeared. For the theory of strongly regular graphs a recent survey is Seidel [63]. For constructions one usually refers to Hubaut [45]. Much of the theory can also be found in Cameron [10] and Cameron and van Lint [14]. A survey on partial geometries describing the state of affairs in 1976 was given by Thas [70]. The main purpose of this paper is to describe briefly what has happened since these earlier surveys appeared.

We assume that the reader is familiar with the theory and with earlier surveys but for the sake of completeness we give several definitions and theorems in this introduction. For details we refer to the literature.

Let $X$ be a finite set. An association scheme with $d$ classes is a pair $\left(X,\left\{R_{0}, R_{1}, \ldots R_{d}\right\}\right)$ such that

(i) $\left\{R_{0}, R_{1}, \ldots, R_{d}\right\}$ is a partition of $X \times X$,

(ii) $R_{0}=\{(x, x) \mid x \in X\}$,

(iii) $R_{i}=R_{i}^{T}(i=0,1, \ldots, d)$,

(iv) there are numbers $p_{i j}^{k}$ (the intersection numbers of the scheme) such that for any pair $(x, y) \in R_{k}$ we have $p_{i j}^{k}=\mid\left\{z \mid(x, z) \in R_{i}\right.$ and $\left.(z, y) \in R_{j}\right\}$. 
We call $n_{i}:=p_{i i}^{0}$ the valency of $R_{i}$ and put $v:=|X|=\Sigma n_{i}$.

A strongly regular graph is an association scheme with 2 classes. The points of $X$ are the vertices of the graph and $\{x, y\}$ is an edge if $(x, y) \in R_{1}$. We shall use the notation

$$
\operatorname{srg}(\nu, k, \lambda, \mu) \text {, where } v=|X|, k=n_{1}, \lambda=p_{11}^{1}, \mu=p_{11}^{2} .
$$

The complement of a srg $(\nu, k, \lambda, \mu)$ is a

$$
\operatorname{srg}(v, l:=v-k-1, v-2 k+\mu-2, v-2 k+\lambda) \text {. }
$$

The $(0,1)$ adjacency matrix of a srg is defined by $A(x, y):=1$ if $\{x, y\}$ is an edge, 0 otherwise. By definition we have

$$
A J=k J, A^{2}+(\mu-\lambda) A+(\mu-k) I=\mu J .
$$

Most of the necessary conditions for the existence of strongly regular graphs were found by studying the eigenvalues of $A$ and properties of the 3-dimensional algebra $A$ of linear combinations of $1, J$, and $A$ (the Bose-Mesner algebra of the $\mathrm{srg}$ ).

Let $E_{j}(0 \leq j \leq 2)$ be a basis of minimal idempotents of $\mathbf{A}$. This algebra is also closed under componentwise multiplication (denoted by O). We define $q_{i j}^{k}$ by

$$
E_{i} \bigcirc E_{j}=v^{-1} \sum_{k=0}^{2} q_{i j}^{k} E_{k}
$$

We point out that Delsarte [26] defined the concept of duality for association schemes (and hence for srg's). A sufficient condition for the existence of a dual is the existence of a regular abelian group of automorphisms. (If a $s r g$ has $v$ vertices, valencies $k$ and $l$, and multiplicities $f$ and $g$, then the dual, if it exists, has $v$ vertices, valencies $f$ and $g$, and multiplicities $k$ and $l$.) Some of the srg's mentioned below were found using duality.

\section{Necessary Conditions}

We list some of the necessary conditions for the existence of a $s r g(v, k, \lambda, \mu)$. We exclude trivial graphs (disconnected graphs and their complements), i.e. we assume $0<\mu<k<v-1$. From the parameters of the complementary graph we see that $v-2 k+\mu-2 \geq 0$ is a necessary condition for the existence of a srg. This kills e.g. $(v, k, \lambda, \mu)=(273,256,240,240)$. 


\section{Integrality Condition}

$A$ has eigenvalue $k$ with multiplicity 1 and two other eigenvalues $r, s(r>s)$ satisfying $x^{2}+(\mu-\lambda) x+(\mu-k)=0$. The multiplicities of these eigenvalues are

$$
f=\frac{-k(s+1)(k-s)}{(k+r s)(r-s)} \text { and } g=\frac{k(r+1)(k-r)}{(k+r s)(r-s)}
$$

and these must clearly be integers. In fact if $f \neq g$ then $r$ and $s$ must be integers. The other case (i.e. $f=g$ ) is called the half-case.

A partial converse to the condition on eigenvalues is the following useful theorem.

THEOREM. A regular connected graph is strongly regular if its adjacency matrix has three eigenvalues.

We shall call a parameter set $(\nu, k, \lambda, \mu)$ feasible if the necessary conditions given above are satisfied.

\section{Partial Geometries}

A partial geometry $\operatorname{pg}(K, R, T)$ is a partial linear space $(X, L)$ with constant line size $K$ (i.e. $|L|=K$ for $L \in \mathbf{L}$ ), such that each point is on $R$ lines and such that given a line $L$ and a point $x \notin L$ there are exactly $T$ lines through $x$ which meet $L$. (Thas [70] uses the notation $s+1=K, t+1=R, \alpha=T)$. If $x \in L, y \in L$ we write $x \sim y$ and say that the points are collinear. The point graph of a partial geometry has the points as vertices and an edge $\{x, y\}$ iff $x \sim y$. The point graph of a $p g(K, R, T)$ is strongly regular (possibly trivial) with parameters

$$
\begin{gathered}
\nu=K\left(1+\frac{(K-1)(R-1)}{T}\right), k=R(K-1), \lambda=(K-2)+(R-1)(T-1), \\
\mu=R T, r=K-1-T, s=-R .
\end{gathered}
$$

It follows that the necessary conditions for the existence of a srg yield necessary conditions for the existence of a $p g$.

If a $\mathrm{srg}$ has parameters such that it could be the point graph of a $p g$ then we call the srg pseudo-geometric and geometric if it is indeed the point graph of a $p g$. We shall see that a pseudo-geometric srg is not necessarily geometric. Bose [4] proved the following theorem.

THEOREM. If a srg is pseudo-geometric corresponding to $p g(K, R, T)$ and if $2 K>R(R-1)+T(R+1)\left(R^{2}-2 R+2\right)$ then the graph is geometric. (In fact it follows that $T=R$ or $T=R-1$.)

The partial geometries can be divided into four classes:

1. A pg with $T=K$ (dually $T=R$ ) is a $2-(v, K, 1)$ design (dual design), 
2. A pg with $T=R-1$ (dually $T=K-1$ ) is a net (transversal design),

3. A pg with $T=1$ is called a generalized quadrangle,

4. If $1<T<\min \{K-1, R-1\}$ then we call the $p g$ proper.

We are only interested in generalized quadrangles (notation: $p g(K, R, 1)=G Q(K-1, R-1))$ and in proper partial geometries.

Constructions for $G Q(s, t)$ are known for $(s, t)=$ resp. $(q, 1),(q, q),\left(q, q^{2}\right),\left(q^{2}, q^{3}\right),(q-1, q+1)$ and the dual sets (here $q$ is a prime power). The proper $p g^{\prime}$ 's described in Thas [70] have

$K=2^{h}-2^{m}+1, R=2^{h}-2^{h-m}+1, T=\left(2^{m}-1\right)\left(2^{h-m}-1\right), 0<m<h$, resp.

$$
K=2^{h}, R=2^{h+m}-2^{h}+2^{m}, T=2^{m}-1 .
$$

For recent new constructions see Section 11 .

In the following table we list the parameters of possible partial geometries with $v<100$ (excluding designs and nets and their duals); (of a dual pair we list the one with $K \leq R$ ).

\begin{tabular}{|c|l|l|l|l|l|l|l|}
\hline$v$ & $k$ & $\lambda$ & $\mu$ & $K$ & $R$ & $T$ & \\
\hline 15 & 6 & 1 & 3 & 3 & 3 & 1 & $G Q(2,2)$, graph is $T(6)^{*}$ \\
27 & 10 & 1 & 5 & 3 & 5 & 1 & $G Q(2,4)$, Schlafli graph \\
28 & 15 & 6 & 10 & 4 & 5 & 2 & $p g$ does not exist, cf. Section 11A \\
40 & 12 & 2 & 4 & 4 & 4 & 1 & $G Q(3,3)$ \\
45 & 28 & 15 & 21 & 5 & 7 & 3 & known, cf. Section 11B \\
64 & 18 & 2 & 6 & 4 & 6 & 1 & $G Q(3,5)$ \\
66 & 45 & 28 & 36 & 6 & 9 & 4 & pg does not exist, cf. Section 11B \\
70 & 42 & 23 & 28 & 7 & 7 & 4 & unknown, several graphs known \\
75 & 32 & 10 & 16 & 5 & 8 & 2 & graph unknown, cf. Section 11H \\
76 & 21 & 2 & 7 & 4 & 7 & 1 & $G Q(3,6)$ does not exist, cf. Section 11C \\
81 & 30 & 9 & 12 & 6 & 6 & 2 & sporadic, cf. Section 11D \\
85 & 20 & 3 & 5 & 5 & 5 & 1 & $G Q(4,4)$ \\
91 & 66 & 45 & 55 & 7 & 11 & 5 & unknown, graph is $T(14)^{*}$, cf. Section 11B \\
95 & 40 & 12 & 20 & 5 & 10 & 2 & graph unknown \\
96 & 35 & 10 & 14 & 6 & 7 & 2 & graph unknown \\
96 & 50 & 22 & 30 & 6 & 10 & 3 & graph unknown \\
\hline
\end{tabular}




\section{Nonexistence Results}

The following six necessary conditions for the existence of a srg will exclude several feasible sets. In each case we give a feasible parameter set which is excluded by that condition but not by any of the other restrictions, showing that these six restrictions are independent.

\section{A. The Half-Case (conference matrices)}

If $f=g$ then we must have $v=4 \mu+1, k=2 \mu, \lambda=\mu-1$. If such a srg exists then there is a conference matrix $C$ of order $v+1$ (cf. Section 7B). It is known (cf. Belevitch [1], van Lint and Seidel [51]) that such a $C$ can only exist if $v$ is the sum of two squares. This kills e.g. $(\nu, k, \lambda, \mu)=(21,10,4,5)$.

\section{B. The Krein Conditions}

It is not difficult to show that all the numbers $q_{i j}^{k}$ must be nonnegative (not necessarily integers). By explicitly computing these numbers we find the four so-called Krein conditions (Krein [50], Scott [60], Higman [39], Delsarte [26]). Two of these are always satisfied. The two nontrivial restrictions are:

$$
\begin{aligned}
& \text { Krein } 1: q_{11}^{1}=\frac{f^{2}}{v}\left(1+\frac{r^{3}}{k^{2}}+\frac{(-1-r)^{3}}{l^{2}}\right) \geq 0, \\
& \text { Krein } 2: q_{22}^{2}=\frac{g^{2}}{v} 1+\frac{s^{3}}{k_{2}}+\frac{(-1-s)^{3}}{l^{2}} \geq 0 .
\end{aligned}
$$

These conditions can be written as

$$
\begin{aligned}
& (r+1)(k+r+2 r s) \leq(k+r)(s+1)^{2} \\
& (s+1)(k+s+2 r s) \leq(k+s)(r+1)^{2} .
\end{aligned}
$$

This kills e.g. $(v, k, \lambda, \mu)=(184,48,2,16)$.

\section{The Absolute Bound}

A suitable linear combination of $I, A$, and $J$ is the Gram matrix of inner products of a spherical two-distance set in $\mathbb{R} f$. Since such a set has at most $\frac{1}{2} f(f+3)$ vectors (cf. Koornwinder [49], Delsarte, Goethals and Seidel [27], Seidel [63]) we have

$$
v \leq \frac{1}{2} f(f+3) \text { and similarly } v \leq \frac{1}{2} g(g+3) \text {. }
$$

This kills e.g. $(\nu, k, \lambda, \mu)=(50,21,4,12)$.

This bound was improved by Neumaier [58] to 


$$
v \leq \frac{1}{2} f(f+1) \text { unless } q_{11}^{1}=0 .
$$

(Graphs with $q_{11}^{1}=0$ or $q_{22}^{2}=0$ are called Smith graphs.) This bound kills e.g. $(\nu, k, \lambda, \mu)=(841,200,87,35)$.

\section{The Claw Bound}

Bose [4] first studied the connection between strongly regular graphs and partial geometries and derived the inequality which we mentioned in Section 2. This idea was extended by Neumaier [57] and after an improvement by Brouwer it obtained the following form:

$$
\text { If } \mu \neq s^{2}, \mu \neq s(s+1) \text { then } 2(r+1) \leq s(s+1)(\mu+1) \text {. }
$$

The idea of the proof is to show that if $r$ is large, then the srg is the point graph of a partial geometry and then applying the absolute bound and the Krein conditions to the line graph of this partial geometry. This kills e.g. $(\nu, k, \lambda, \mu)=(2058,242,91,20)$.

\section{E. The Case $\mu=1$}

As was remarked by Bose and Dowling [5], if $\mu=1$ then the set of neighbours of a point is a union of $(\lambda+1)$ - cliques. Hence $(\lambda+1) \mid k$. This kills e.g. $(v, k, \lambda, \mu)=(726,29,4,1)$. The total number of $(\lambda+2)$-cliques is $v k /(\lambda+1)(\lambda+2)$ which must be an integer. This kills e.g. $(v, k, \lambda, \mu)=(209,16,3,1)$.

\section{F. The Case $\mu=2$}

It was observed by Brouwer and Neumaier [8] that if $\mu=2$ then the set of neighbours of a point carries the structure of a partial linear space of girth at least five. This implies that if $k<\frac{1}{2} \lambda(\lambda+3)$ then $(\lambda+1) \mid k$. This kills e.g. $(v, k, \lambda, \mu)=(736,42,8,2)$. Slightly more information is available which also kills $(v, k, \lambda, \mu)=(1944,67,10,2)$. Besides the above six necessary conditions we mention a few other results.

\section{G. The Mu-Bound}

By combining various bounds Neumaier [57] proved that

$$
\mu \leq s^{3}(2 s+3)
$$

with equality iff $v=\frac{1}{2} f(f+3)$. This is a very useful bound, but since it follows as a consequence of the bounds mentioned above it cannot lead to new nonexistence results. 


\section{H. Sporadic Nonexistence Results}

For two graphs nonexistence was shown by ad hoc arguments. The first of these has $(\nu, k, \lambda, \mu)=(49,16,3,6)$, (Bussemaker et al [9]) and in this case part of the argument is a computer search. The second set is $(v, k, \lambda, \mu)=(57,14,1,4)$, (Wilbrink and Brouwer [73]). The proof depends on counting arguments for subgraphs. More specifically it is shown that both the assumption that the graph contains a 15coclique and the contrary lead to a contradiction. These two parameter sets were the smallest for which the existence question could not be settled by any of the other conditions. At present the smallest open case is $(v, k, \lambda, \mu)=(65,32,15,16)$ corresponding to a conference matrix of order 66 .

\section{Restrictions on Subsets}

Although we are primarily interested in constructions we shall have occasion to use some results of a more theoretical nature. First of all we have the so called Hoffman bounds on the size of a clique or a coclique in the graph:

THEOREM 1. Let $C$ be a clique in a strongly regular graph with parameters $(v, k, \lambda, \mu, r, s)$. Then

$$
|C| \leq 1+k /(-s)
$$

and equality holds iff each point outside $C$ is adjacent to exactly $\mu /(-s)$ points of $C$.

COROLLARY. Suppose a graph is pseudogeometric with parameters $(\boldsymbol{K}, R, T)$. If we can find cliques of size $K(=1+k /(-s))$ such that each edge is in exactly one such clique then the graph is geometric (and $T=\mu /(-s))$.

THEOREM 2. Let $C$ be a coclique in a strongly regular graph with parameters $(v, k, \lambda, \mu, r, s)$. Then

$$
|C| \leq \frac{v \cdot(-s)}{k-s}
$$

and equality holds iff each point outside $C$ is adjacent to exactly $-s$ points of $C$.

Sometimes the Cvetkovic bound is sharper:

THEOREM 3. The size of a coclique $C$ of a graph $G$ cannot exceed the number of nonnegative (nonpositive) eigenvalues of $G$.

THEOREM 4. (Haemers [35]). Suppose a coclique $C$ in a strongly regular graph $G$ meets both previous bounds, i.e., 


$$
|C|=g=v .(-s) /(k-s) \text {. }
$$

Then the subgraph of $G$ induced by the complement of $C$ is strongly regular.

A second group of results (Cameron, Goethals \& Seidel [11]) concerns the case of equality in the absolute bound or the Krein condition. Let us quote only one theorem.

THEOREM 5. Let $G$ be a strongly regular graph with $q_{11}^{1}=0$ or $q_{22}^{2}=0$. Then for every vertex $x$ the subconstituents $\Gamma(x)(=\{y \mid y \sim x\})$ and $\Delta(x)(=\{y \mid y \neq x, y+x\})$ are both strongly regular.

\section{Uniqueness Results}

\section{A. Triangular Graphs}

The graphs with as vertices the pairs from $\{1,2, \ldots, n\}$ joined by an edge if they have an element in common are the triangular graphs $T(n)$. They have as parameters $v=\left(\begin{array}{l}n \\ 2\end{array}\right), \quad k=2(n-2)$, $\lambda=n-2, \mu=4$. Graphs with these parameters are unique for $n \neq 8$. For $n=8$ apart from $T(8)$ there exist exactly three other graphs with the same parameters, the so-called Chang graphs. All four graphs are switching equivalent (cf. Chang [16,17], Connor [22], Hoffman [42], Seidel [63]).

\section{B. Lattice Graphs}

The lattice graph $L(n)$ has as vertices the points $(x, y) \in\{1,2, \ldots \ldots\}^{2}$, joined by an edge if the first or second coordinates are the same. They have $v=n^{2}, k=2(n-1), \lambda=n-2$, $\mu=2$. Graphs with these parameters are unique for all $n \neq 4$. For $n=4$ there is one graph besides $L(4)$, the so-called Shrikhande graph. These two are switching equivalent (cf. Shrikhande [65]).

\section{Small Graphs}

All graphs on $v<25$ vertices are uniquely determined by their parameters except for the parameter set $(16,6,2,2)$ referred to above. Apart from triangular graphs and lattice graphs (and their complements) this set includes the Paley graphs of order 5,9,13 and 17 and the Clebsch graph with parameters $(v, k, \lambda, \mu)=(16,5,0,2)$. 


\section{Sporadic Graphs} exists.

For the following parameter sets it is known that a unique graph

\begin{tabular}{|c|c|c|c|c|c|c|c|c|c|}
\hline$v$ & $k$ & $\lambda$ & $\mu$ & $r$ & $s$ & $f$ & 8 & name & unique by \\
\hline 16 & 5 & 0 & 2 & 1 & -3 & 10 & 5 & Clebsch & $\begin{array}{l}\text { Seidel [61], } \\
\text { Clatworthy [18] }\end{array}$ \\
\hline 27 & 10 & 1 & 5 & 1 & -5 & 20 & 6 & Schläfli $(G Q(2,4))$ & Seidel [61] \\
\hline 50 & 7 & 0 & 1 & 2 & -3 & 28 & 21 & Hoffman-Singleton & $\begin{array}{l}\text { Hoffman \& } \\
\text { Singleton [43] }\end{array}$ \\
\hline 56 & 10 & 0 & 2 & 2 & -4 & 35 & 20 & Sims-Gewirtz & Gewirtz $[31,32]$ \\
\hline 77 & 16 & 0 & 4 & 2 & -6 & 55 & 21 & co sub HiS $(S(3,6,22))$ & Brouwer [6] \\
\hline 100 & 22 & 0 & 6 & 2 & -8 & 77 & 22 & Higman-Sims & Gewirtz [32] \\
\hline 112 & 30 & 2 & 10 & 2 & -10 & 90 & 21 & sub $\operatorname{McL}(G Q(3,9))$ & $\begin{array}{l}\text { Cameron, } \\
\text { Goethals \& Seidel [11] }\end{array}$ \\
\hline 162 & 56 & 10 & 24 & 2 & -16 & 140 & 21 & co sub $\mathrm{McL}$ & $\begin{array}{l}\text { Cameron, } \\
\text { Goethals, \& Seidel [11] }\end{array}$ \\
\hline 275 & 112 & 30 & 56 & 2 & -28 & 252 & 22 & McLaughlin & Goethals \& Seidel [34] \\
\hline
\end{tabular}

\section{E. Geometric Graphs}

Cameron, Goethals and Seidel [11] showed that a pseudogeometric graph corresponding to $G Q\left(q, q^{2}\right)$ is always geometric. This allows one to show that the graph is unique as soon as uniqueness of the generalized quadrangle is known.

\section{F. Regular Two-Graphs}

A two-graph is a set $\Omega$ of triples from a set $X$ such that every 4 subset of $X$ contains an even number of triples from $\Omega$. For the strong connection between srg's and two-graphs and the theory of switching we refer to Seidel [62]. The regular two-graphs on 16, 28 and on 276 vertices are unique (Taylor [69], Goethals and Seidel [34]) and each has a transitive group of automorphisms. By isolating a point through switching and then removing it, this shows that the Schläfli and McLaughlin graphs are unique. It also shows that graphs with parameters $(v, k, \lambda, \mu)=(16,6,2,2)$ or $(28,12,6,4)$ or $(276,135,78,54)$ are switching equivalent. For $v=16$ see Section $5 B$; for $v=28$ see Section $5 A$; for $v=276$ only one graph is known. 


\section{Strongly Regular Graphs found by Merging Classes in an Association Scheme}

\section{A. The Johnson Scheme}

In the Johnson scheme $\left(\begin{array}{l}n \\ 3\end{array}\right)$ call two triples adjacent if they have exactly one point in common. When $n=7$ or $n=10$ this defines a srg. (For $n=7$ this is the line graph of $P G(3,2)$; for $n=10$ we find parameters $v=120, k=63, \lambda=30, \mu=36$.)

In the Johnson scheme $\left(\begin{array}{l}n \\ 4\end{array}\right)$ call two quadruples adjacent if they have either zero or three points in common. When $n=9$ or $n=11$ this defines a srg. Parameters: $v=126, k=25, \lambda=8, \mu=4$ resp. $v=330, k=63, \lambda=24, \mu=9$. In the Johnson scheme $\left(\begin{array}{c}12 \\ 4\end{array}\right)$ call two quadruples adjacent if they have either zero or two points in common. We get a $\operatorname{srg}(495,238,109,119)$. (These graphs were found by Mathon [55a].)

\section{B. The Hamming Scheme}

In the Hamming scheme $4^{n}$ call two vectors adjacent if their distance is odd. This yields a srg with parameters $v=2^{2 n}$, $k=2^{2 n-1}+\epsilon 2^{n-1}, \lambda=\mu=2^{2 n-2}+\epsilon 2^{n-1}$ with $\epsilon=(-1)^{n-1}$. If $n$ is even this graph has the same parameters as the line graph of an orthogonal array (or transversal design) $O A\left(2^{n}, 2^{n-1}\right)$, but for $n>2$ these are nonisomorphic. In all cases these graphs have the parameters of the graphs in Hubaut's family $C_{12}$.

In the Hamming scheme $3^{n}$ call two two distinct vectors $i$-th associates iff their distance is congruent to $i(\bmod 3),(i=1,2,3)$. This yields a 3-class association scheme. If $n$ is even then merging any two of the three classes yields a srg. Parameters: write $n=2 m$, then $v=3^{2 m}$.

(a) adjacent iff distance $=0(\bmod 3)$ (but nonzero):

$$
\begin{aligned}
k & =3^{2 m-1}+(-1)^{m} \cdot 2 \cdot 3^{m-1}-1, \\
\lambda & =3^{2 m-2}+(-1)^{m} \cdot 2 \cdot 3^{m-1}-2, \\
\mu & =3^{2 m-2}+(-1)^{m} \cdot 3^{m-1} .
\end{aligned}
$$

(b) adjacent iff distance $\equiv 1(\bmod 3)$

resp. adjacent iff distance $\equiv 2(\bmod 3)$ : 


$$
\begin{aligned}
k & =3^{2 m-1}-(-1)^{m} \cdot 3^{m-1}, \\
\lambda & =3^{2 m-2}, \\
\mu & =4 \cdot 3^{2 m-2}+(-1)^{m} \cdot 3^{m-1}-2
\end{aligned}
$$

(cf. Kageyama, Saha and Das [46])

A simpler proof than the one in [46] can be given by using the fact that the eigenvalues of the Hamming scheme are values of Krawtchouk polynomials. Well known relations for these polynomials show that merging leads to a situation with only three different eigenvalues and then the theorem in Section 1 can be applied. (The weight of a vector $(\bmod 3)$ is the value of the quadratic form $Q(x)=\Sigma x_{i}^{2}$. From this, one immediately sees that the two graphs under $b$ ) are isomorphic and that the graph $a$ ) is isomorphic to the appropriate one in Hubaut's family $C_{12}$.)

\section{The Cyclotomic Scheme}

Let $q=p^{(e-1) t}$ where $p$ is a prime, $t$ is even, $e$ is a prime $>2$ and $p$ is primitive $(\bmod e)$. Call two elements $x, y$ of the field $I F_{q} j$-th associates $(0<j \leq e)$ if $x-y=\alpha^{e i+j}$ for some $i$, where $\alpha$ is a fixed primitive element of $I F_{q}$. Now any single relation of this association scheme gives us a (rank 3 ) strongly regular graph of negative Latin square type, and the union of $u$ classes gives a srg with parameters

$$
\begin{gathered}
v=q, k=\frac{q-1}{e} . u, \lambda=\left(u^{2} q-3 u e+u^{2}-(e-u)(e-2 u) q^{\frac{1}{2}}\right) / e^{2}, \\
\mu=\left(u^{2} q-u e+u^{2}+u(e-2 u) q^{\frac{1}{2}}\right) / e^{2} \\
r=\left(-1+q^{1 / 2}\right) u / e, s=r-q^{1 / 2}, \\
f=(q-1)(e-u) / e, g=k
\end{gathered}
$$

(cf. van Lint and Schrijver [52]).

More generally one might take $q$ a prime power, $e$ a divisor of $q-1$ and call two elements $x, y$ of $I F_{q} j$-th associates $(0<j \leq e)$ if $x-y=\alpha^{e i+j}$ for some $i$. Sometimes a union of classes yields a srg. Examples where this works:

$q=81, e=8, \nu=81, k=30, \lambda=9, \mu=12$

(vanLint \& Schrijver [52], Mesner [56]),

$q=243, e=11: \nu=243, k=22, \lambda=1, \mu=2$ 
(Berlekamp, vanlint and Seidel [3]), $\nu=243, k=110, \lambda=37, \mu=60$

(Delsarte [26], dual of previous), $q=256, e=15, \nu=256, k=68, \lambda=12, \mu=20$

(Brouwer, unpublished).

\section{Other Examples}

Let $q$ be a power of two. The group $\operatorname{PSL}(2, q)$ acts as a rank $\frac{1}{2} q$ group on the $\frac{1}{2} q(q-1)$ exterior lines of a hyperoval in $P G(2, q)$. The corresponding association scheme has valencies $n_{0}=1$ and $n_{i}=q+1$ $\left(1 \leq i \leq \frac{1}{2} q-1\right)$. Under the larger group $P \Sigma L(2, q)$ some of these classes merge, and H.D.L. Hollmann [44] observed that when $q=16$ one obtains a 3-class association scheme with valencies $1,17,34,68$ such that merging relations $R_{1}$ and $R_{2}$ yields a srg with parameters $v=120, k=51, \lambda=18, \mu=24$. (Most likely this graph is isomorphic to the $q=4$ member of the Metz family treated in Section 7A.)

$P \Gamma L(2,8)$ acts 2 -transitively (on the Ree unital with parameters $S(2,4,28)$ ) on 28 points. $P G L(2,8)$ acts as a rank 4 group, giving a 3class association scheme with parameters $v=28, n_{1}=n_{2}=n_{3}=9$,

$p_{i j}^{1}=\left(\begin{array}{llll}0 & 1 & 0 & 0 \\ 1 & 2 & 4 & 2 \\ 0 & 4 & 2 & 3 \\ 0 & 2 & 3 & 4\end{array}\right), p_{j k}^{i}=p_{j+1, k+1}^{i+1}$ for $\{i, j, k\} \subset\{1,2,3\},($ addition mod 3$)$.

The unital is the collection of 4-sets with a proper colouring, i.e., two disjoint edges in each of the three relations. A second association scheme with the same parameters can be obtained from the previous one by 'switching' with respect to a block of the unital; its group of automorphisms is elementary abelian of order 8 . These two are the only schemes with these parameters (Hollman [44]). The eigenvalues of these schemes are 


$$
P=\left(\begin{array}{cccc}
1 & 9 & 9 & 9 \\
1 & \lambda_{1} & \lambda_{2} & \lambda_{3} \\
1 & \lambda_{3} & \lambda_{1} & \lambda_{2} \\
1 & \lambda_{2} & \lambda_{3} & \lambda_{1}
\end{array}\right)
$$

where the $\lambda_{i}$ are the solutions of $\lambda^{3}+\lambda^{2}-9 \lambda-1=0$ (cf. Mathon [53]).

Now consider the direct product of two such schemes. This is a 15-class association scheme with relations

$$
R_{i j}=\left\{\left(x x^{\prime}, y y^{\prime}\right) \mid(x, y) \in R_{i} \text { and }\left(x^{\prime}, y^{\prime}\right) \in R_{j}\right\}
$$

and eigenvalues $\mu_{i} \mu_{j}$ where $\mu_{i}\left(\mu_{j}\right)$ runs through the eigenvalues of $R_{i}\left(R_{j}\right)$. One finds that $R_{11} \cup R_{22} \cup R_{33}$ has eigenvalues $3 n^{2}=243$, $k\left(\lambda_{1}+\lambda_{2}+\lambda_{3}\right)=-9, \quad \lambda_{1}^{2}+\lambda_{2}^{2}+\lambda_{3}^{2}=19 \quad$ and $\lambda_{1} \lambda_{2}+\lambda_{1} \lambda_{3}+\lambda_{2} \lambda_{3}=-9$ so that we have found a strongly regular graph.

Similarly, $R_{11} \cup R_{22} \cup R_{33} \cup R_{10} \cup R_{20} \cup R_{30}$ has eigenvalues 270,18 and -10 , and $R_{11} \cup R_{22} \cup R_{33} \cup R_{10} \cup R_{20} \cup R_{30} \cup R_{01} \cup R_{02} \cup R_{03}$ has eigenvalues 297,17 , and -11 .

Thus we have found strongly regular graphs with parameters

$\begin{array}{cccccccc}\nu & k & \lambda & \mu & r & s & f & g \\ 784 & 243 & 82 & 72 & 19 & -9 & 243 & 540 \\ 784 & 270 & 98 & 90 & 18 & -10 & 270 & 513 \\ 784 & 297 & 116 & 110 & 17 & -11 & 297 & 486\end{array}$

This construction is due to $R$. Mathon (private communication) and applies more generally to pseudo-cyclic 3-class association schemes. The graphs produced have the parameters of Latin square graphs and have new parameters only when $v$ is not a prime power. Write $p_{1 j}^{1}=(1 t-r-s-1 s r)$ and $n_{j}=t$. Now a pseudo-cyclic association scheme $\operatorname{SPC}(r, s, t)$ on $v=3 t+1$ points produces three strongly regular graphs on $v^{2}$ points with $k=3 t^{2}+3 i t, r=2 t+1-i, s=-t-i$ $(i=0,1,2)$. Now Hollmann constructed a $\operatorname{SPC}(50,58,165)$ on 496 points (see [44]) so that we find new graphs on 246016 vertices.

The symmetric group $S_{9}$ acts as a rank 5 group on the 280 partitions of a 9-set into three triples. (The valencies are 1, 27, 36, 54 and 162.) The graph $\left(X, R_{4}\right)$ is strongly regular with parameters $(280,162,96,90)$ (Mathon and Rosa [55b]). 


\section{Strongly Regular Graphs found using Classical Groups and Geometries}

\section{A. A Construction by R. Metz}

Let $Q$ be a nonsingular elliptic quadric in $P G(5, q)$. Let $p$ be a point and let $\Pi$ be a hyperplane such that $p$ is not on $Q$ or on $\Pi$. Let $V:=\{x \in \Pi \mid p x$ meets $Q$ in two points $\}$. Let $\mathbf{L}:=\{l \mid l$ is a line of $\Pi$ such that the plane $\langle p, l\rangle$ meets $Q$ in two intersecting lines\}. Let $I:=\{(x, l) \mid x \in l\}$. Then $(V, \mathbf{L}, I)$ is a semipartial geometry with parameters $s+1=q, t+1=q^{2}+1, \alpha=2, \mu=2 q(q-1)$. (For a definition of semipartial geometry see Debroey and Thas [25].) The point graph of this geometry is strongly regular with parameters:

$v=\frac{1}{2} q^{2}\left(q^{2}-1\right), k=(q-1)\left(q^{2}+1\right), \lambda=(q-1)(q+2), \mu=2 q(q-1)$,

$r=q-1, s=-(q-1)^{2}, f=\frac{1}{2}(q-2)(q+1)\left(q^{2}+1\right), g=\frac{1}{2} q\left(q^{2}+1\right)$.

The above description seems to be due to Hirschfeld and Thas; the description given by Metz being the following (for a discussion of the isomorphism of these descriptions, see Debroey [24]): Let $Q$ be a nonsingular quadric in $P G(4, q)$. Let $V$ be the set of hyperplanes meeting $Q$ in an elliptic quadric. If $x, y \in V$ then let $x \sim y$ if the corresponding elliptic quadrics are tangent. (Now lines can be defined as bundles of mutually tangent quadrics. ) This representation of $\Omega(5, q)$ is rank 3 for $q=4,8$.

\section{B. A Variant by Brouwer and Wilbrink}

Let $Q$ be a nonsingular quadric in $P G(4, q)$. Let $V$ be the set of hyperplanes meeting $Q$ in a hyperbolic quadric. If $x, y \in V$ then let $x \sim y$ if the corresponding hyperbolic quadrics are tangent (i.e., if $x \cap y \cap Q$ consists of two intersecting lines). This defines a srg with parameters

$$
\begin{gathered}
\nu=\frac{1}{2} q^{2}\left(q^{2}+1\right), \quad k=(q+1)\left(q^{2}-1\right), \\
\lambda=(q-1)(3 q+2), \quad \mu=2 q(q+1), \\
r=q^{2}-2 q-1, \quad s=-q-1, f=\frac{1}{2} q(q+1)^{2}, \\
g=\frac{1}{2}(q-2)(q+1)\left(q^{2}+1\right) .
\end{gathered}
$$

Again one has lines (bundles of mutually tangent quadrics), and $s+1=q, t+1=(q+1)^{2}$. This time one does not obtain a 
semipartial geometry, but a structure with the property that given a point $p$ and a line $l$ such that $p \notin l$ there are 0 or 2 or $q$ points on $l$ joined to $p$. For odd $q$ the complementary graph is pseudo-geometric corresponding to a $p g\left(\frac{1}{2}\left(q^{2}+1\right), q(q-2), \frac{1}{2}(q-1)^{2}-1\right)$, and it is indeed possible to find cliques of size $\frac{1}{2}\left(q^{2}+1\right)$ in this graph: Let $E$ be an elliptic line (i.e., $E \cap Q=\varnothing$ ) and define $C_{E}:=\left\{x \in V \mid E^{\downarrow} \subset x\right.$ or $\left.E \subset x\right\}$. Then $C_{E}$ is a clique of size $\frac{1}{2}\left(q^{2}+1\right)$. The number of elliptic lines $E$ is $\frac{1}{2} q^{3}(q-1)\left(q^{2}+1\right)$ and for $q>3$ each $C_{E}$ determines $E$ uniquely, so that we have far too many cliques for a partial geometry, and it turns out to be impossible to choose an appropriate subcollection. For $q=3$ however, each $C_{E}$ belongs to ten distinct lines $E$ and we find 27 sets $C_{E}$ yielding a partial geometry $p g(5,3,1)$. Of course this is the well known generalized quadrangle $G Q(4,2)$, thus illustrating the sporadic isomorphism $\operatorname{PSU}(4,2) \cong \Omega(5,3)$.

\section{Generalization of the Preceding Two Constructions}

Wilbrink observed that the preceding two constructions in fact work in all dimensions. Thus: Let $Q$ be a nonsingular quadric in $P G(2 m, q)$. Let $V$ be the set of hyperplanes meeting $Q$ in a hyperbolic (elliptic) quadric. Let $\epsilon=+1(-1)$. If $x, y \in V$ then let $x \sim y$ if the corresponding quadrics are tangent (i.e., if $Q \cap x \cap y$ is degenerate). This defines a $s r g$ with parameters

$$
\begin{gathered}
v=\frac{1}{2} q^{m}\left(q^{m}+\epsilon\right), k=\left(q^{m}-\epsilon\right)\left(q^{m-1}+\epsilon\right), \\
\lambda=2\left(q^{2 m-2}-1\right)+\epsilon q^{m-1}(q-1) \\
\mu=2 q^{m-1}\left(q^{m-1}+\epsilon\right), \\
r, s=-\epsilon q^{m-1}-1 \text { and } \epsilon q^{m-1}(q-2)-1
\end{gathered}
$$

\section{Another Construction by Wilbrink}

Let $Q$ be a nonsingular quadric in $P G(2 m, 5)$. Let $V$ be the set of nonisotropic points $x$ such that $x^{1}$ meets $Q$ in a $\left\{\begin{array}{c}\text { hyperbolic } \\ \text { elliptic }\end{array}\right\}$ quadric. Let $\epsilon= \pm 1$. If $x, y \in V$ then $x \sim y$ if $x \perp y$. This defines a srg with parameters 


$$
\begin{gathered}
v=\frac{1}{2} 5^{m}\left(5^{m}+\epsilon\right), k=\frac{1}{2} \cdot 5^{m-1}\left(5^{m}-\epsilon\right), \\
\lambda=\frac{1}{2} \cdot 5^{m-1}\left(5^{m-1}+\epsilon\right), \mu=\frac{1}{2} \cdot 5^{m-1}\left(5^{m-1}-\epsilon\right), \\
\quad r, s=2 \epsilon \cdot 5^{m-1} \text { and }-\epsilon \cdot 5^{m-1} .
\end{gathered}
$$

\section{E. A construction by D. Taylor}

Let $H$ be a hermitian form on the projective plane $P G\left(2, q^{2}\right)$, where $q$ is an odd prime power. Let $U=\{x \mid H(x, x)=0\}$ be the corresponding unital. Then $|U|=q^{3}+1$. One can define a regular two-graph on $U$ by taking the triples $\{x, y, z\}$ for which $-H(x, y) H(y, z) H(z, x)$ is a nonsquare in $G F\left(q^{2}\right)$. Isolating a point $\infty$ by switching and then deleting it yields a srg with parameters

$$
\begin{gathered}
v=q^{3}, k=2 \mu=\frac{1}{2}(q-1)\left(q^{2}+1\right), \lambda=\frac{1}{4}(q-1)^{3}-1, \\
r=\frac{1}{2}(q-1), s=-\frac{1}{2}\left(q^{2}+1\right), f=(q-1)\left(q^{2}+1\right), \quad g=q(q-1) .
\end{gathered}
$$

(This is Hubaut's family $C_{20}$.) Note that collinear triples of the unital are members of the two-graph, so that after switching $\Lambda\{\infty\}$ has become a clique, for each line $l$ passing through $\infty$. Thus our graph has a partition into cliques of size $q$ (achieving the Hoffman bound) so that any point outside such a clique has exactly $\frac{1}{2}(q-1)$ neighbours on it. Consequently, if we take the union of any $\frac{1}{2}\left(q^{2}+1\right)$ of these cliques we get a regular subgraph of degree $\frac{1}{4}(q-1)\left(q^{2}+3\right)$, and adding a new point $\Omega$ and switching yields a srg with parameters

$$
\begin{gathered}
v=q^{3}+1, k=\frac{1}{2} q\left(q^{2}+1\right), \lambda=\frac{1}{4}(q-1)\left(q^{2}+3\right), \mu=\frac{1}{4}(q+1)\left(q^{2}+1\right), \\
r=\frac{1}{2}(q-1), s=-\frac{1}{2}\left(q^{2}+1\right), f=(q-1)\left(q^{2}+1\right), g=q^{2}-q+1 .
\end{gathered}
$$

(It is conceivable that there is another srg in the switching class of this regular two-graph. It would have $k=\frac{1}{2} q^{2}(q-1), \lambda=\frac{1}{4}\left(q^{2}+1\right)(q-3), \quad \mu=\frac{1}{4}(q-1)\left(q^{2}-1\right)$. For $q=3$ such a graph does not exist (it violates the absolute bound). For $q=5$ such a graph was constructed by Goethals (see Section 10B on the Hoffman-Singleton graph). For larger $q$ such graphs are unknown. Note that for $q=3^{2 n+1}$ the Ree groups have a doubly transitive representation on $q^{3}+1$ points, yielding a regular two-graph with the 
same parameters as the one associated with $P \Gamma U\left(3, q^{2}\right)$ discussed above. This again leads to $\operatorname{srg}$ 's on $q^{3}$ points (and possibly $q^{3}+1$ ?).)

\section{F. A Construction by W.M. Kantor}

Let $V$ be a vector space over $G F(q)$, equipped with a symplectic, orthogonal or unitary geometry. A spread of $V$ is a family $\Sigma$ of maximal totally isotropic or singular subspaces which partitions the set $P$ of totally isotropic or singular points.

Construct a graph with as vertices the set $\Omega$ of all hyperplanes of members of $\Sigma$, and join two distinct vertices $X, Y$ when $X \cap Y^{\perp} \neq 0$. This graph is strongly regular and has the same parameters as the graph with vertices $P$ and as edges the pairs of perpendicular points. This construction and variations of it produce large numbers of nonisomorphic strongly regular graphs (see Kantor [46]).

\section{Combinatorial Constructions}

\section{A. A Construction by Haemers}

Let $q=2^{n}$ and let $K$ be a hyperoval in $P G(2, q)$ (considered as hyperplane at infinity of $A G(3, q)$ ). Let $V$ be the set of lines in $A G(3, q)$ having a point of $K$ as their point at infinity, and for $x, y \in V$ set $x \sim y$ if $x$ and $y$ meet (in an affine point). This defines a strongly regular graph with parameters

$$
\begin{gathered}
v=q^{2}(q+2), k=q(q+1), \lambda=\mu=q, \\
r=q, s=-q, f=\left(\frac{1}{2} q+1\right)\left(q^{2}-1\right), g=\frac{1}{2} q(q+1)^{2} .
\end{gathered}
$$

(This is Hubaut's family $C_{18}$ ) (In fact, with the pencils of lines as Lines this is a $G Q(q+1, q-1)$.) Now partition the $q+2$ parallel classes of lines in an arbitrary way into $\frac{1}{2} q+1$ pairs: $k=\cup_{i=1}^{q / 2+1}\left\{u_{i}, v_{i}\right\}$. Redefine adjacency so that for each $i$ two lines with direction $u_{i}$ (resp. $v_{i}$ ) become adjacent iff the plane they span passes through the point $v_{i}$ (resp. $u_{i}$ ), while no line with direction $u_{i}$ will be adjacent to a line with direction $v_{i}$. All other adjacencies are unchanged. This defines a new srg with parameters

$$
\begin{gathered}
v=q^{2}(q+2), k=q^{2}+q-1, \lambda=q-2, \mu=q, \\
r=q-1, s=-(q+1), f=\frac{1}{2}(q+2)\left(q^{2}+q-1\right), g=\frac{1}{2} q\left(q^{2}+q-1\right) .
\end{gathered}
$$




\section{B. A Construction by Mathon}

A symmetric conference matrix of order $n$ is a matrix $C$ with zero diagonal and entries \pm 1 elsewhere such that $C=C^{T}$ and $C C^{T}=(n-1) 1$. A necessary condition for the existence of such a matrix is $n \equiv 2(\bmod 4)$. A srg with half-case parameters $(v-1=2 k=4 \mu)$ exists iff there exists a symmetric conference matrix of order $v+1$. (Given such a matrix, normalize it such that the first row and first column contain 0 and +1 's only and delete row and column. If the resulting matrix is $B$ then $A:=\frac{1}{2}(B+J-1)$ is the adjacency matrix of a srg and the process may be reversed.) Symmetric conference matrices of order $n$ are known in the following cases (cf. Wallis et al [72], Appendix C):

(i) $n=q+1, q$ a prime power, $q \equiv 1(\bmod 4)$,

(ii) $n=(h-1)^{2}+1, h$ the order of a skew Hadamard matrix,

(iii) $n=(m-1)^{a}+1, m$ the order of a symmetric conference matrix, $a$ any nonnegative integer.

Mathon gave a new recursive construction for symmetric conference matrices proving that if $q=4 t-1$ is a prime power and there exists a symmetric conference matrix of order $q+3$, then there exists a symmetric conference matrix of order $q^{2}(q+2)+1$. (See Mathon [54] or Seidel and Taylor [64].) This produces e.g. $\operatorname{srg}(45,22,10,11)$.

\section{A construction by Goethals and Seidel}

Let $N$ be the $V$ by $B$ incidence matrix of a Steiner system $S(2, K, V)$. Let $H$ be a Hadamard matrix of order $R+1$; normalize it so that its first column contains ones only and delete this column. Call the resulting $R+1$ by $R$ matrix $L$. (Now $J L=0$.) Let $P$ be the $(R+1) V$ by $B$ matrix obtained from $N$ by replacing each row by $R+1$ rows: replace the $R$ one entries of a row by the $R$ columns of $L$ and the zero entries of the row by zero columns. Let $P P^{T}=R I+A$. Then $(A+R I)(A-(V+K-1) I)=0$ and $A J=-R J$. (See [61].) It follows that $\frac{1}{2}(J-I-A)$ is the adjacency matrix of a $s r g$ with parameters

$$
\begin{gathered}
v=(R+1) V, k=\frac{1}{2}(v-1+R), \lambda=\frac{1}{4}(R-1)(V+K+2), \mu=\frac{1}{4}(R+1)(V+K), \\
r=\frac{1}{2}(R-1), s=-\frac{1}{2}(V+K), f=v-1-g, g=B=R V / K .
\end{gathered}
$$

(These are the parameters of a pseudo-geometric graph corresponding to $p g\left(R+1, \frac{1}{2}(V+K), \frac{1}{2}(R+1)\right)$, but e.g. $p g(4,5,2)$ does not exist so that these graphs cannot always be geometrical.) 


\section{Regular Symmetric Hadamard Matrices with Constant Diagonal}

Suppose $H$ is a regular symmetric Hadamard matrix of order $n$ with constant diagonal, i.e., $H H^{T}=n l, H=H^{T}, H J=a J$, $h_{i j} \in\{ \pm 1\}, h_{i i}=\epsilon(1 \leq i, j \leq n)$. (Then clearly $\left.n=a^{2}\right)$.) Then $\frac{1}{2}(J-I-H+\epsilon I)$ is the adjacency matrix of a srg with parameters $v=n, k=\frac{1}{2}(n-1-a+\epsilon), \lambda=\frac{1}{4}(n-2 a)-(1-\epsilon), \mu=\frac{1}{4}(n-2 a)$.

Obviously, by replacing $H$ by $-H$ if necessary, we may assume $a>0$. Also, if $n>1$ then $4 \mid n$ so that we may write $a=2 t$. This yields for the parameters:

$$
\text { If } \begin{array}{rlrl}
\epsilon=+1: v & =4 t^{2}, & k=2 t^{2}-t, & \lambda=\mu=t^{2}-t, \\
r, s= \pm t, & f=2 t^{2}-t, & g=2 t^{2}+t-1 .
\end{array}
$$

(These are the parameters of a pseudo Latin square graph $L_{t}(2 t)$.)

$$
\text { If } \begin{aligned}
\epsilon=-1: v=4 t^{2}, & k=2 t^{2}-t-1, \quad \lambda=t^{2}-t-2, \quad \mu=t^{2}-t \\
r=t-1, & s=-t-1, \quad f=2 t^{2}+t, \quad g=2 t^{2}-t-1
\end{aligned}
$$

(These are the parameters of a negative Latin square graph $N L_{t}(2 t)$.) (And conversely, graphs with these parameters yield regular symmetric Hadamard matrices with constant diagonal.) In view of the above we are interested in the set of pairs $(n, \epsilon)$ for which such a matrix (with positive row sums) exists. Let us call this set RSHCD. There is one recursive construction:

$$
(m, \delta),(n, \epsilon) \in R S H C D \rightarrow(m n, \delta \epsilon) \in R S H C D .
$$

The following direct constructions are known:

(i) $(4, \pm 1),(36, \pm 1) \in R S H C D$,

(ii) If there exists a Hadamard matrix of order $m$ then $\left(m^{2}, 1\right) \in R S H C D$,

(iii) If both $a-1$ and $a+1$ are odd prime powers then $\left(a^{2}, 1\right) \in R S H C D$,

(iv) If $a+1$ is a prime power and there exists a symmetric conference matrix of order a then $\left(a^{2}, 1\right) \in R S H C D$,

(v) If there is a set of $t-2$ mutually orthogonal latin squares of order $2 t$ (i.e., a transversal design $T[t ; 2 t])$ then $\left(4 t^{2}, 1\right) \in R S H C D$.

(iv) Suppose we have a Steiner system $S(2, K, V)$ with $V=K(2 K-1)$. If we form the blockgraph and add an isolated point we get a graph in the switching class of a regular two-graph. The 
corresponding Hadamard matrix is symmetric with constant diagonal, but not regular (as claimed in [72], p. 454). If moreover this Steiner system is invariant under a regular abelian group of automorphisms (which necessarily has orbits on the blocks of sizes $V$, $V$ and $2 K-1$ ) then by switching with respect to a block orbit of size $V$ we obtain a srg with parameters

$$
v=4 K^{2}, \quad k=V=K(2 K-1), \quad \lambda=\mu=K(K-1)
$$

showing that $\left(4 K^{2}, 1\right) \in R S H C D$. Steiner systems $S(2, K, K(2 K-1))$ are known for $K=3,5,6,7$ or $2^{t}$, but only for $K=2,3,5,7$ are systems known that have a regular abelian group of automorphisms. Thus we find $(196,1) \in R S H C D$. [The required switching set also exists when the design is resolvable: take the union of $K$ parallel classes. Resolvable designs are known for $K=3$ or $2^{t}$.]

(Most of the constructions in this section are due to Goethals \& Seidel [33]; for more details see Wallis [72], Section 5.3.)

\section{Rank 3 Representations of Finite Groups}

The rank 3 permutation representations of the finite classical groups have been determined by Kantor and Liebler [48]. Their main result is the following: (we quote)

"THEOREM 1. Let $M$ be one of the groups $S p(2 m-2, q)$, $\Omega^{ \pm}(2 m, q), \Omega(2 m-1, q)$ or $S U(m, q)$ for $m \geq 3$ and $q$ a prime power. Let $M \unlhd G$ with $G / Z(M) \leq A u t(M / Z(M))$. Assume that $G$ acts as a primitive rank 3 permutation group on the set $X$ of cosets of a subgroup $K$ of $G$. Then (at least) one of the following holds up to conjugacy under $A u t(M / Z(M))$.

(i) $X$ is an $M$-orbit of singular (or isotropic) points.

(ii) $X$ is an $M$-orbit of maximal totally singular (or isotropic) subspaces and $M=S p(4, q), S U(4, q), S U(5, q), \Omega^{-}(6, q), \Omega^{+}(8, q)$, or $\Omega^{+}(10, q)$.

(iii) $X$ is any $M$-orbit of nonsingular points and $M=S U(m, 2)$, $\Omega^{ \pm}(2 m, 2), \Omega^{ \pm}(2 m, 3)$ or $\Omega(2 m-1,3)$.

(iv) $X$ is either orbit of nonsingular hyperplanes of $M=\Omega(2 m-1,4)$ or $\Omega(2 m-1,8)$ (where $G=\Gamma O(2 m-1,8)$ in the latter case).

(v) $M=S U(3,3), K \cap M=P S L(3,2)$.

(vi) $M=S U(3,5), K \cap M=3 . A_{7}$.

(vii) $M=S U(4,3), K \cap M=4 . P S L(3,4)$.

(viii) $M=S p(6,2), K=G_{2}(2)$. 
(ix) $M=\Omega(7,3), K \cap M=G_{2}(3)$.

(x) $M=S U(6,2), K \cap M=3 \cdot P S U(4,3) \cdot 2$.

THEOREM 2. Let $M=\operatorname{PSL}(n, q) \leq G \leq A u t M$. Assume that $G$ acts as a primitive rank 3 permutation group on the set $X$ of cosets of a subgroup $K$ of $G$. Then (at least) one of the following occurs up to conjugacy under Aut $M$.

(i) $X$ is the set of lines for $M, n \geq 4$.

(ii) $M=\operatorname{PSL}(2,4) \cong \operatorname{PSL}(2,5),|X|=\left(\begin{array}{l}5 \\ 2\end{array}\right)$,

$$
\begin{aligned}
& M=\operatorname{PSL}(2,9) \cong A_{6},|X|=\left(\begin{array}{l}
6 \\
2
\end{array}\right) \\
& G=\operatorname{PSL}(4,2) \cong A_{8},|X|=\left(\begin{array}{l}
8 \\
2
\end{array}\right), \text { or } \\
& G=\operatorname{PrL}(2,8),|X|=\left(\begin{array}{l}
9 \\
2
\end{array}\right) .
\end{aligned}
$$

(iii) $M=P S L(3,4), M \cap K=A_{6}$.

(iv) $M=P S L(4,3), M \cap K=P S p(4,3)$.

The reader is cautioned that isomorphisms between, and automorphisms of, the various groups allow many different ways of viewing some of these cases. Specifically, the isomorphisms $\operatorname{PSp}(4, q) \cong \Omega(5, q)$ $P S U(4, q) \cong P \Omega^{-}(6, q) \quad P S L(4, q) \cong P \Omega^{+}(6, q)$ and $P S U(4,2) \cong P S p(4,3)$ lead to numerous representations under (1(i)). Similarly, a triality automorphism of $P \Omega^{+}(8, q)$ can be applied to (1(iii)) and a polarity automorphism can be applied to (2(i)).".

Not each of these permutation representations was mentioned by Hubaut, but it seems that no new graphs are obtained. (For the parameters in case 1 (iv) and arbitrary $q$ see Section $7 \mathrm{C}$ - Wilbrink's construction.)

F. Zara [75] was so kind as to supply us with the parameters of two rank 3 representations of the Fischer groups not mentioned by Hubaut:

$$
\begin{array}{ccccccccc}
\multicolumn{1}{c}{\nu} & \multicolumn{1}{c}{k} & \multicolumn{1}{c}{l} & \lambda & \mu & \bar{\lambda} & \bar{\mu} & G & G_{0} \\
14080 & 10920 & 3159 & 8408 & 8680 & 918 & 648 & F i_{22} & O^{+}(7,3) \\
137632 & 109200 & 28431 & 86600 & 86800 & 6030 & 5832 & F i_{23} & \left(O^{+}(8,3)\right)^{\prime} . S_{3}
\end{array}
$$

(Here $O^{+}(n, 3)$ is the subgroup of the orthogonal group generated by the reflections $x+(x, a) a$, where $(a, a)=1$.) 


\section{Some Sporadic Graphs}

\section{A. The Higman-Sims Family}

Almost all sporadic graphs are constructed in some way from the Steiner systems $S(5,6,12)$ or $S(5,8,24)$ or from the binary or ternary Golay code. (In other words, all graphs described in this section can be found somewhere in the Leech lattice.) Let us start with graphs derived from the Steiner system $S(3,6,22)$.

(i) $(\nu, k, \lambda, \mu)=(77,16,0,4)$.

Take as vertices the blocks of the (unique) design $S(3,6,22)$ and join two vertices by an edge when the corresponding blocks are disjoint. This produces a strongly regular graph with the stated parameters. (It is unique - Brouwer [6].)

(ii) $(v, k, \lambda, \mu)=(56,10,0,2)$ - the Gewirtz graph.

The preceding graph contains a coclique of size 21 (i.e. meeting both the Hoffman bound and the Cvetkovic bound) namely all blocks containing a fixed point. Deleting this coclique produces a strongly regular graph with the stated parameters. It was first found by C.C. Sims (unpublished) and shown to be unique by Gewirtz [31]. Adding the identity matrix to an adjacency matrix of this graph we obtain a symmetric block design 2-(56,11,2) (a biplane). (This always works when $\mu=\lambda+2$; when $\mu=\lambda$ then the graph itself already gives a symmetric block design. We shall soon see an example where the graph is derived from a block design (with polarity).)

(iii) $(v, k, \lambda, \mu)=(100,22,0,6)$ - the Higman-Sims graph.

Take a symbol $\infty$, the 22 points and the 77 blocks of $S(3,6,22)$. Join $\infty$ to the 22 points, join a point to the blocks containing it, and join two blocks when they are disjoint. This produces a strongly regular graph with the stated parameters. Its uniqueness was shown by Gewirtz [32]. The simple group of Higman and Sims is an index two subgroup of its automorphism group.

(iv) $(v, k, \lambda, \mu)=(231,30,9,3)-$ the Cameron graph.

Take as vertices the $\left(\begin{array}{c}22 \\ 2\end{array}\right)=231$ unordered pairs from the point set of $S(3,6,22)$. Join two vertices when the pairs are disjoint and their union is contained in a block. This produces a graph with the given parameters (cf. [11]). One may introduce lines as triples of pairwise disjoint pairs with as their union a block of the design; the geometry thus obtained is a gamma space in Higman's terminology, i.e. given a point $x$ not on a line $L$, then $x$ is joined to 0,1 or all points of $L$ (as observed by [8a] and [10a]). 
(v) $(\nu, k, \lambda, \mu)=(176,49,12,14)$.

(Perhaps this graph does not belong in this section - for instance it does not have 2 as an eigenvalue.) Let $x_{0}$ and $x_{1}$ be two fixed points in the point set of $S(5,8,24)$. Construct a symmetric design with as points the blocks containing $x_{0}$ but not $x_{1}$, and as blocks the blocks containing $x_{1}$ but not $x_{0}$. A point $B$ is incident with a block $B^{\prime}$ iff $\left|B \cap B^{\prime}\right| \in\{0,4\}$. It is straightforward to check that this produces a symmetric $2-(176,50,14)$ design. (This design was first constructed by $G$. Higman [40]; this representation is due to Margaret Smith [67].) This design has exactly two polarities, one with 176 and one with 80 fixed points (Sims [66], Brouwer [7]). By using a polarity of the first kind we can write the incidence matrix $A$ as a symmetric matrix with ones on the diagonal, and $A-I$ is the adjacency matrix of the required srg. The graph has $S_{8}$ as group of automorphisms. Uniqueness is unknown. For a detailed discussion of G. Higman's geometry and its relations to the Hoffman-Singleton graph, see Broue and Enguehard [5a].

\section{B. The Hoffman-Singleton Family}

Moore showed that a graph with valency $k$ and girth $2 d+1$ can have at most $1+k+k(k-1)+\ldots+k(k-1)^{d-1}$ vertices. (A similar bound can be obtained for the case for even girth.) Graphs that meet this bound are called Moore graphs. For $d=2$ we find $v \leq k^{2}+1$, and one sees immediately that the graph must be strongly regular when equality holds (with $\lambda=0$ and $\mu=1$ ). The parameter set $\left(k^{2}+1, k, 0,1\right)$ is admissible only for $k=2,3,7$ and 57 and we find for $k=2$ the pentagon, for $k=3$ the Petersen graph $T(5)^{*}$, for $k=7$ the Hoffman-Singleton graph to be discussed below; while the case $k=57$ is still open.

(i) $(\nu, k, \lambda, \mu)=(50,7,0,1)$ - the Hoffman-Singleton graph.

Let $X=\{\infty\} \cup \mathbf{P} \cup \mathbf{B}$ be the point set of the Higman-Sims graph as constructed above. Let $B_{0}$ be a block not containing $\Omega$ of a design $S(4,7,23)$ with point set $\mathbf{P} \cup\{\Omega\}$ that has our design $(\mathbf{P}, \mathbf{B})$ as its derived design at the point $\Omega$. Then $B_{0}$ induces a partition of the point set of the Higman-Sims graph as follows:

$$
X=\left(\{\infty\} \cup B_{0} \cup\left\{B \in \mathbf{B}|| B \cap B_{0} \mid=1\right\}\right) \cup\left(\mathbb{P} \backslash B_{0} \cup\left\{B \in \mathbf{B}|| B \cap B_{0} \mid=3\right\}\right) .
$$

One can check that :

(a) The induced subgraph on each of the two sets into which $X$ is partitioned is (isomorphic to) the Hoffman-Singleton graph.

(b) Any partition of the Higman-Sims graph into two HoffmanSingleton graphs arises in this way. 
(The Hoffman-Singleton graph was first constructed and proved to be unique by Hoffman and Singleton [43]. The observation that the Higman-Sims graph contains two copies of the Hoffman-Singleton graph seems to be due to Sims [66].)

Conversely, one may define the Higman-Sims graph in terms of the Hoffman-Singleton graph: The Hoffman-Singleton graph contains 100 cocliques of size 15 . Two such cocliques intersect in $0,3,5,8$, or 15 points. This defines a 4-class association scheme (relations : $R_{0}$ : identity, $R_{1}, R_{2}, R_{3}, R_{4}$ : intersection of cardinality $8,5,3,0$ respectively) with parameters $v=100, n_{0}=1, n_{1}=15, n_{2}=42, n_{3}=35, n_{4}=7$

$$
\begin{aligned}
\left(p_{0 j}^{i}\right)=I_{5},\left(p_{1 j}^{i}\right) & =\left(\begin{array}{ccccc}
0 & 15 & 0 & 0 & 0 \\
1 & 0 & 14 & 0 & 0 \\
0 & 5 & 0 & 10 & 0 \\
0 & 0 & 12 & 0 & 3 \\
0 & 0 & 0 & 15 & 0
\end{array}\right), \quad\left(p_{2 j}^{i}\right)=\left(\begin{array}{ccccc}
0 & 0 & 42 & 0 & 0 \\
0 & 14 & 0 & 28 & 0 \\
1 & 0 & 35 & 0 & 6 \\
0 & 12 & 0 & 30 & 0 \\
0 & 0 & 36 & 0 & 6
\end{array}\right), \\
\left(p_{3 j}^{i}\right) & =\left(\begin{array}{ccccc}
0 & 0 & 0 & 35 & 0 \\
0 & 0 & 28 & 0 & 7 \\
0 & 10 & 0 & 25 & 0 \\
1 & 0 & 30 & 0 & 4 \\
0 & 15 & 0 & 20 & 0
\end{array}\right), \quad\left(p_{4 j}^{i}\right)=\left(\begin{array}{ccccc}
0 & 0 & 0 & 0 & 7 \\
0 & 0 & 0 & 7 & 0 \\
0 & 0 & 6 & 0 & 1 \\
0 & 3 & 0 & 4 & 0 \\
1 & 0 & 6 & 0 & 0
\end{array}\right) \\
P & =\left(\begin{array}{cccccc}
1 & 15 & 42 & 35 & 7 \\
1 & -15 & 42 & -35 & 7 \\
1 & 0 & -3 & 0 & 2 \\
1 & 5 & 2 & -5 & -3 \\
1 & -5 & 2 & 5 & -3
\end{array}\right), Q=\left(\begin{array}{ccccc}
1 & 1 & 56 & 21 & 21 \\
1 & -1 & 0 & 7 & -7 \\
1 & 1 & -4 & 1 & 1 \\
1 & -1 & 0 & -3 & 3 \\
1 & 1 & 16 & -9 & -9
\end{array}\right)
\end{aligned}
$$

This scheme is imprimitive: $\left(X, R_{4}\right)$ is the disjoint union of two Hoffman-Singleton graphs. The graph $(X, R)$ is a regular thin near octagon, and is uniquely determined by its parameters. Since $\left(X, R_{1} \cup R_{4}\right)$ has only three eigenvalues it is strongly regular, and we find the Higman-Sims graph again. One may construct a system of three 'linked' Hoffman-Singleton graphs on 150 vertices by taking the vertices and each of two sets of 50 cocliques; join vertices in the obvious way. Now on each of the three sets of 100 points this graph induces a Higman-Sims graph.

For later use we mention two other constructions of the HoffmanSingleton graph.

(ii) The first construction is due to $\mathbf{N}$. Robertson [59].

Let $P_{\alpha}\left(\alpha \in I F_{5}\right)$ be five pentagons and let $Q_{\beta}\left(\beta \in I F_{5}\right)$ be five 
pentagrams. Number the points of the $P_{\alpha}$ and $Q_{\beta}$ with elements of $I F_{5}$ such that points in $P_{\alpha}$ are adjacent iff their labels differ by one, and in $Q_{\beta}$ iff their labels differ by two. Draw an edge between point $i$ of $P_{\alpha}$ and point $j$ of $Q_{\beta}$ whenever $j=i+\alpha \beta$. This produces the HoffmanSingleton graph.

COROLLARY: It is possible to split the point set of the HoffmanSingleton graph into two halves such that the induced subgraph on each of these halves is the union of five pentagons. The union of two pentagons from different halves induces a Petersen graph. Each pentagon uniquely determines a split so that there are 126 splits. Each split determines 25 Petersen graphs and any two splits determine a unique Petersen graph. In this way we find a $2-(126,6,1)$ design on splits and Petersen graphs: this design turns out to be the classical unital in $P G\left(2,5^{2}\right)$ so that we can identify the automorphism group of the Hoffman-Singleton graph as $P \sum U\left(3,5^{2}\right)$. (Benson and Losey [2].) Two split-halves meet in 10 or in 15 points; if we fix a point $x$ and consider the graph on the splits, where two splits are joined if their halves containing $x$ meet in 10 points, then we find a srg with parameters $(v, k, \lambda, \mu)=(126,50,13,24)$. Another construction of this graph is given under (vii).

(iii) The second construction uses the well known fact that it is possible to identify the lines of $P G(3,2)$ with the triples formed out of seven elements in such a way that intersecting lines correspond to triples that have exactly one element in common.

Let $C$ be the set of points and $D$ the set of lines of $P G(3,2)$, so that $|C|=15$ and $|E|=35$. Join a line to the points on it and join two lines when the corresponding triples are disjoint. This produces the Hoffman-Singleton graph.

(iv) $(v, k, \lambda, \mu)=(175,72,20,36)$ and a new partial geometry.

On the 175 edges of the Hoffman-Singleton graph (abbreviated Ho-Si) we have a 3-class association scheme (with relations $R_{0}=$ identity, $R_{1}$ = intersecting, $R_{2}=$ together in a pentagon, $R_{3}=$ the remainder), and Aut(Ho-Si) acts as a rank 4 group. The parameters are

$\left(p_{0 j}^{i}\right)=I_{4},\left(p_{1 j}^{i}\right)\left(\begin{array}{cccc}0 & 12 & 0 & 0 \\ 1 & 5 & 6 & 0 \\ 0 & 1 & 6 & 5 \\ 0 & 0 & 4 & 8\end{array}\right)\left(p_{2 j}^{i}\right)=\left(\begin{array}{llll}0 & 0 & 72 & 0 \\ 0 & 6 & 36 & 30 \\ 1 & 6 & 20 & 45 \\ 0 & 4 & 36 & 32\end{array}\right),\left(p_{3 j}^{i}\right)=\left(\begin{array}{cccc}0 & 0 & 0 & 90 \\ 0 & 0 & 30 & 60 \\ 0 & 5 & 45 & 40 \\ 1 & 8 & 32 & 49\end{array}\right)$.

The graph corresponding to $R_{1}$ is distance transitive of diameter 3 with diagram 


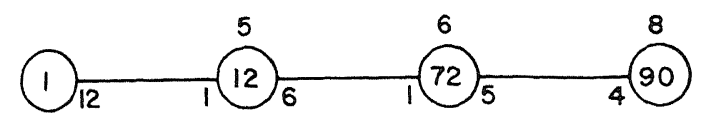

and eigenvalues $12^{1}, 7^{28}, 2^{21},(-2)^{125}$; it is the line graph of Ho-Si. (In fact, any distance regular graph with parameters $i(12,6,5 ; 1,1,4)$ is necessarily this graph: $\mu=1$ implies that each edge lies in a unique 7 clique; there are 50 such 7-cliques and one sees immediately that the graph on the 7-cliques is $\mathrm{Ho}-\mathrm{Si}$.)

The graph corresponding to $R_{2}$ is strongly regular with parameters $(\nu, k, \lambda, \mu)=(175,72,20,36)$ and eigenvalues $72^{1}, 2^{153},(-18)^{21}$.

W.H. Haemers [36] observed that it carries the structure of a partial geometry $\mathrm{pg}(5,18,2)$ :

We have to find 630 lines of size 5, i.e. 6305 -cliques. But a 5-clique is a set of 5 edges of $\mathrm{Ho}-\mathrm{Si}$, pairwise in a pentagon. These 5 edges induce a Petersen subgraph in Ho-Si. Ho-Si contains 525 Petersen graphs and each Petersen graph contains 6 matchings, i.e. our graph contains 3150 5-cliques and we must choose one-fifth of them in such a way that any pentagon determines a unique 'special' Petersen graph. to this end let $C$ be a 15-coclique and write the point set of $\mathrm{Ho}-\mathrm{Si}$ as $C \cup D$. Observe the following:

(1) The 35 points in $D$ each have 3 neighbours in $C$, and we may identify $C$ and $D$ with the points and lines of the projective space $P G(3,2)$ as above.

(2) Of the 1260 pentagons, 630 have one point in $C$ and 630 have two points in $C$. Let $\mathbf{P}_{i}=\{$ pentagons with $i$ points in $C\}(i=1,2)$.

(3) $A u t(H o-S i)_{C} \cong A_{7}$ acts transitively on each set of 630 pentagons.

(4) There is a 1-1 map I, invariant under this group, from $\mathbf{P}_{2}$ onto $\mathbf{P}_{1}$ such that each $P_{2} \cup \amalg P_{2}$ induces a Petersen graph $P$ with the property that the three points in $P \cap C$ have a common neighbour. (For: if $P_{2} \cap C=\{x, y\}$ then the line $L=x y z$ is a vertex of $P_{2}$, and $P_{2}$ together with the edge $L z$ determines a Petersen graph $P$. Now $P \cap C=\{x, y, z\} \subset \Gamma(L)$.)

(5) There are 105 such special Petersen graphs; each is a union $P_{1} \cup P_{2}$ in six ways. Each of them contains 6 matchings, and 
thus we find the required 630 lines.

The partial geometry thus constructed has $A_{7}$ as its group of automorphisms.

(v) $(v, k, \lambda, \mu)=(630,85,20,10)$.

Given the existence of a $p g(5,18,2)$ it follows by duality that $p g(18,5,2)$ exists. Its point graph has the indicated parameters.

(vi) $(\nu, k, \lambda, \mu)=(176,70,18,24)$ and $(\nu, k, \lambda, \mu)=(176,90,38,54)$.

The graph found under (iv) has $k=2 \mu$, so that adjoining an isolated point produces a regular two-graph on 176 vertices. This two-graph contains strongly regular graphs with the stated two parameter sets in its switching class. In order to construct such graphs we have to indicate suitable switching sets - in this case regular subgraphs of the 175 graph with 70 (resp. 90) points and valency 18 (resp.38). For the first set there is a natural choice: in the representation of Ho-Si as $C \cup D$ used above there are 70 edges in $D$, and $R_{2}$ has valency 18 on this set of 70 edges. For the second set Haemers indicated the following possibility: Take the union of 18 pairwise disjoint 5-cliques. Since these cliques attain the Hoffman bound, each point outside a clique is adjacent to two points inside, so this union has valency $4+17.2=38$. Such disjoint 5-cliques do exist: in fact from N. Robertson's reprsentation of Ho-Si one sees immediately that there exist 25 pairwise disjoint 5-cliques.

(vii) $(\nu, k, \lambda, \mu)=(126,50,13,24)$.

If we remove a vertex $x$ and its neighbours from $\mathrm{Ho}-\mathrm{Si}$, we get a graph on 42 vertices and 126 edges. J.-M. Geothals observed that the subgraph of the 175-graph (iv) induced on these 126 edges is strongly regular with the stated parameters. The direct verification is somewhat tedious; it is easier to show that the graph has only three eigenvalues. To this end let us quote a very useful theorem from [35]:

THEOREM. Suppose

$$
A=\left(\begin{array}{ll}
A_{11} & A_{12} \\
A_{12}^{*} & A_{22}
\end{array}\right)
$$

is hermitean of size $n$. Suppose $A$ has just two eigenvalues, that is

$$
\lambda_{1}(A)=\cdots=\lambda_{f}(A)>\lambda_{f+1}(A)=\cdots=\lambda_{n}(A)
$$

for some $f, 1 \leq f<n$. Let $n_{1}$ and $n_{2}$ be the sizes of $A_{11}$ and $A_{22}$ respectively. Then $\lambda_{i}\left(A_{22}\right)=$ 


$$
\begin{array}{ll}
\lambda_{1}(A)+\lambda_{n}(A)-\lambda_{f-i+1}\left(A_{11}\right) & \text { for } \max \left\{1, f+1-n_{1}\right\} \leq i \leq \min \left\{f, n_{2}\right\}, \\
\lambda_{1}(A) & \text { for } 1 \leq i \leq f+1-n_{1}, \\
\lambda_{n}(A) & \text { for } f<i \leq n_{2} .
\end{array}
$$

This theorem can be applied to $(-1,+1,0)$ adjacency matrices of strong graphs in the switching class of a regular two-graph (i.e., matrices satisfying $\left.\left(A-\rho_{1} I\right)\left(A-\rho_{2} I\right)=0\right)$ :

COROLLARY. Suppose some strong graph in the switching class of a regular two-graph with $v$ vertices and eigenvalues $\rho_{1}, \rho_{2}\left(\rho_{1}>\rho_{2}\right)$ with multiplicities $\mu_{1}, \mu_{2}$ has a subgraph with $u \geq \mu_{1}$ vertices and eigenvalues $\lambda_{1}, \ldots, \lambda_{u}$, where $\lambda_{1}=\rho_{1}$ and $\lambda_{2}=\cdots=\lambda_{\mu_{1}}$. Then the subgraph induced on the remaining $v-u$ vertices is a strong graph with two eigenvalues: $\rho_{1}+\rho_{2}-\lambda_{2}$ with multiplicity $\mu_{1}-1$ and $\rho_{2}$ with multiplicity $v-u-\mu_{1}+1$.

Application: Adding an isolated vertex to the 175 graph and switching with respect to the seven edges on $x$ we get a graph containing Ho-Si in the switching class of the two-graph. The $(-1,+1,0)$ eigenvalues of the two-graph are $\rho_{1}=35$ and $\rho_{2}=-5$ (with $\mu_{1}=22, \mu_{2}=154$ ) and of Ho-Si 35,5 and -5 with multiplicities 1,21 and 28 , so that the corollary produces a strong graph on 126 vertices. Observing that it is regular of valency 50 shows that we found the right graph.

\section{The McLaughlin Family}

(i) $(v, k, \lambda, \mu)=(275,112,30,56)$ - the McLaughlin graph.

Consider the 23 points and 253 blocks of the (unique) Steiner system $(X, B)$ with parameters $S(4,7,23)$. Define a graph on 276 vertices with $X$ as coclique by joining a point to the blocks containing it and joining two blocks whenever they intersect in one point. This defines a (graph in the switching class of a) regular two-graph on 276 points. (For a matrix proof see Seidel [62]; for the interpretation as a set of equiangular lines in the Leech lattice see Taylor [69].) Its automorphism group is Conway .3. Isolating a vertex by switching and then deleting it produces a rank 3 graph on 275 vertices with McLaughlin's simple group as group of automorphisms. Graph and two-graph are unique ([34]).

From the definition it is clear that the 276-graph constructed above contains the Higman-Sims graph (switched w.r.t. one vertex). The $(-1,+1,0)$ eigenvalues of the two-graph are $\rho_{1}=55$ and $\rho_{2}=-5$ with multiplicities $\mu_{1}=23, \mu_{2}=253$, and of the Higman-Sims graph $55,15,-5$ with multiplicities $1,22,77$ (respectively) so that (by the corollary in $B$ (vii)) we find a strongly regular graph on 176 vertices, with as vertices the blocks of the residual design $S(4,7,23) \cup(3,6,22)$ 
and as edges the pairs of blocks meeting in one point. The parameters are $(v, k, \lambda, \mu)=(176,70,18,24)$, the same graph as we found above in $B$ (vi). This time we immediately see that its group of automorphisms is the Higman-Sims simple group. The McLaughlin graph is extremal in several respects: it has $g=22$ and thus satisfies the absolute bound with equality; it has $q_{22}^{2}=0$ so it satisfies the Krein condition with equality; its complement has $s=-3$ and $\mu=81$ and hence satisfies the $\mu$-bound with equality.

(ii) $(\nu, k, \lambda, \mu)=(162,56,10,24)$ and $(\nu, k, \lambda, \mu)=(112,30,2,10)$.

Cameron, Goethals and Seidel [11] proved that $q_{22}^{2}=0$ implies that both subconstituents are strongly regular. Applying this to the previous graph produces two graphs with the stated parameters.

Let us give direct descriptions of these graphs. Under PSL $(3,4)$ the projective plane $P G(2,4)$ contains three orbits of Fano subplanes. Take as vertices the points, the lines, and one orbit of Fano subplanes. Then $v=21+21+120=162$. Let the points and the lines be two 21-cocliques; join a point to a line when they are not incident; join a point to a Fano plane when they are incident; join a line to a Fano plane when it meets it in three points and join two Fano planes when they meet in one point. This produces the unique $\operatorname{srg}(162,56,10,24)$.

Let $x_{0}$ and $x_{1}$ be two fixed points in the point set of $S(4,7,23)$. Take as vertices the 112 blocks that contain exactly one of these points. Join two blocks that contain the same fixed point when this is the only point they have in common. Join a block containing $x_{0}$ to a block containing $x_{1}$ when they have three points in common. This produces the unique $\operatorname{srg}(112,30,2,10)$, the point graph of the generalized quadrangle $G Q(3,9)$. From the construction it is clear that the point set of this graph can be split into two halves such that the graph induced on each half is the Gewirtz graph. Just as we saw for the Hoffman-Singleton graph, it is possible to construct a system of three 'linked' Gewirtz graphs on 168 vertices (starting with three fixed points $x_{0}, x_{1}$ and $x_{2}$ in $S(5,8,24)$ and taking as vertices those blocks that contain exactly two of these points, joining blocks $B$ and $B^{\prime}$ iff $\left|B \cap B^{\prime}\right|+2\left|B \cap B^{\prime} \cap\left\{x_{0}, x_{1}, x_{2}\right\}\right|=6$ ) such that any two Gewirtz graphs induce the point graph of $G Q(3,9)$. This graph is the point graph of a partial linear space with lines of size 6 .

(iii) $(\nu, k, \lambda, \mu)=(276,140,58,84)$.

Goethals and Seidel [34] showed (by explicitly indicating a switching set) that the regular two-graph on 276 vertices contains a $\mathrm{srg}$ with the stated parameters in its switching class. Uniqueness is unknown.

(iv) $(v, k, \lambda, \mu)=(243,110,37,60)$ - the Delsarte graph. 
In the course of their uniqueness proof for the 276-point regular twograph Goethals and Seidel found that it contained in its switching class a graph with $11 K_{3}$ (the disjoint union of 11 triangles) as induced subgraph, and with each of the remaining vertices having exactly one neigbour on each triangle. From the theorem in $B$ (vii) one finds that the graph on the remaining 243 vertices has $(-1,+1,0)$ eigenvalues $49^{22}, 22^{1},(-5)^{220}$ and hence is the required graph. This graph was first constructed by Delsarte from the ternary Golay code (see below).

\section{The Golay Codes and the Hill Cap}

(i) $(v, k, \lambda, \mu)=(243,22,1,2)$ - the Berlekamp-vanLint-Seidel graph.

In [3] three distinct constructions are given (one for each author). The easiest is the following: take as vertices the $3^{5}=243$ cosets of the perfect ternary Golay code, and join two vertices when the corresponding cosets have representatives differing by a vector of weight one. This graph has a Delsarte dual (since it has a regular abelian group of automorphisms)-this dual has parameters $(v, k, \lambda, \mu)$ $=(243,110,37,60)$; we saw it earlier as a subgraph of the 276 -point regular two-graph.

(ii) $(\nu, k, \lambda, \mu)=(2048,276,44,36)$

$$
(\nu, k, \lambda, \mu)=(2048,759,310,264) \text {. }
$$

and

Take the $2^{11}=2048$ cosets of even weight of the extended binary Golay code as vertices, and join two cosets when they have representatives differing by a vector of weight two. This yields a $\operatorname{srg}(2048,276,44,36)$. (An equivalent but more complicated description can be found in Hubaut [45]. This graph was first constructed by J.H. Conway and M.S. Smith. Taking all $2^{12}$ cosets and joining two cosets when they have distance one yields a regular thin near octagon on 4096 vertices in which this graph is the $d=2$ subgraph on one of the halves of the bipartition.) The Delsarte dual of this graph is a $\operatorname{srg}(2048,759,310,264)$. It was first constructed by Goethals and Seidel, who gave the following description: Take the $2^{11}=2048$ cosets of $\{\underline{0}, \underline{1}\}$ in the extended binary Golay code and join two cosets when they have Hamming distance 8.

(iii) $(v, k, \lambda, \mu)=(1288,792,476,504)$.

The complement of the second graph in (ii) is a $\operatorname{srg}(2048,1288,792,840)$. Its first subconstituent is the required graph.

(iv) $(\nu, k, \lambda, \mu)=(729,112,1,20)$ - the Games graph.

There is a unique 56-cap in $P G(5,3)$ (that is a set of 56 points such that any line meets it in at most two points), cf. Hill [41]. R. Games observed that if we take as vertices the points of $A G(6,3)$ and join two 
vertices when the line through the points meets the hyperplane at infinity in a point of the cap, then we obtain a $\operatorname{srg}(729,112,1,20) .(c f .[9 a],[30]$.

\section{Recent Results on Partial Geometries}

\section{A. $p g(4,5,2)$ does not exist.}

In [19] F. de Clerck classifies the pseudo-geometric graphs corresponding to a partial geometry with $T=K-2$. An interesting result is the nonexistence of a $p g(4,5,2)$. In Section $5 \mathrm{~A}$ we observed that there are exactly four non-isormorphic $\operatorname{srg}(28,15,6,10)$ : the complement of the triangular graph $T(8)$ and the Chang graphs. For each of these one can assume that the $s r g$ is geometric and arrive at a contradiction. For the triangular graph the proof is as follows. Let the points be the unordered pairs $(x, y)$ from $\{1,2, \ldots, 8\}$. A line is a partition of $\{1,2, \ldots, 8\}$. W.l.o.g. we can take (12) (34) (56) (78) as a line and in fact we can give all the lines through (12) w.1.o.g. This leaves two possibilities for the line through (13) and (24) and then it turns out to be impossible to give the remaining lines through (13). The other proofs are similar.

\section{B. Partial Geometries from $T(n)^{*}$}

$$
\text { If } \left.n \text { is even then } \operatorname{srg}\left(\begin{array}{l}
n \\
2
\end{array}\right),\left(\begin{array}{c}
n-2 \\
2
\end{array}\right),\left(\begin{array}{c}
n-4 \\
2
\end{array}\right)\left(\begin{array}{c}
n-3 \\
2
\end{array}\right)\right) \text { is pseudo- }
$$

geometric, corresponding to $p g\left(\frac{1}{2} n, n-3, \frac{1}{2} n-2\right)$. We saw above that it is possible to settle the question of existence by hand if $n=8$. For $n=10$ a $p g(5,7,3)$ is known. It can be constructed as follows. Consider a hyperoval $\Sigma$ in $P G(2,8)$. Take the 45 secants of $\Sigma$ as new points and the points not on $\Sigma$ as new lines. The new points and lines are the $p g$. If we number the points of $\Sigma$ from 1 to 10 then the five secants through a point not on $\Sigma$ yield a partition of $\{1,2, \ldots, 10\}$ corresponding to our representation above.

It was shown by $R$. Mathon [55] that there is one other $p g(5,7,3)$. (See also [29a].) At present an exhaustive computer search is being carried out by C.W.H. Lam for the next case, i.e. $p g(6,9,4)$. If this partial geometry does not exist then it will follow that a possible projective plane of order 10 does not have a hyperoval and hence does not have an extension.

Added in proof: After 183 days of computation the exhaustive search was completed and no $p g$ was found [50a]. Thus, assuming correctness of the programs involved, no $p g(6,9,4)$ and no $S(3,12,112)$ 
exists.

\section{C. $p g(4,7,1)$ does not exist}

It is not known whether a $\operatorname{srg}(76,21,2,7)$ exists or not but Dixmier and Zara $[28,29]$ have shown that a $p g(4,7,1)$ cannot exist. We give a slight modification of their proof. Suppose the partial geometry exists.

(i) Let $x+y, \operatorname{tr}(x, y):=\{z \mid z \sim x, z \sim y\}$,

$$
\Delta(x, y):=\{z \mid z+x, z+y\} \text {. Then }|\operatorname{tr}(x, y)|=7,|\Delta(x, y)|=39 \text {. }
$$

(ii) For $i=0,1, \ldots 7$ let $K_{i}$ be the subset of $\Delta(x, y)$ consisting of points which are collinear with $i$ points in $\operatorname{tr}(x, y)$ and let $n_{i}:=\left|K_{i}\right|$.

(iii) Elementary counting yields $\quad \Sigma n_{i}=39, \quad \sum i . n_{i}=105$, $\Sigma\left(\begin{array}{l}i \\ 2\end{array}\right) n_{i}=105$.

(iv) Let $p$ and $q$ be collinear points in $\Delta$ such that the line $L$ through $p$ and $q$ does not meet $t r(x, y)$. Then for the other two points $a$ and $b$ on $L$ we have $x \sim a, y \sim b$ and the lines through $x$ and $a$, resp. $y$ and $b$ meet $\operatorname{tr}(x, y)$ in different points. It follows that if $p \in K_{i}$ then $q \in K_{5-i}$. From this we find that $(7-i) n_{i}=(2+i) n_{5-i}$ and hence $n_{6}=0$. From (iii) we then find $n_{0}=n_{5}=n_{7}=0$, $n_{1}=4, n_{2}=12, n_{3}=15, n_{4}=8$.

(v) Let $p \in K_{1}$ and let $p \sim z \in \operatorname{tr}(x, y)$. From (iv) it follows that the other two points on the line $p z$ are both in $K_{4}$. There are six lines through $p$ not meeting $\operatorname{tr}(x, y)$ and on each we have a point in $K_{4}$ by (iv). Since $n_{4}=8$ we see that each point in $K_{1}$ is adjacent to each point in $K_{4}$, contradicting $\mu=7$.

D. The Sporadic Geometry $p g(6,6,2)$

In [52] van Lint and Schrijver describe a partial geometry with $K=R=6, T=2$ which is at present one of the two sporadic partial geometries. The corresponding srg is of the type described in Section 5C. The easiest description is as follows. The 81 vertices are the codewords of the $(5,4)$ linear code $C$ over $I F_{3}$ with $(1,1,1,1,1)$ as parity check. Two vertices are joined by an edge if their distance is $\equiv 2(\bmod 3)$. The six codewords $(0,0,0,0,0)$, $(1,2,2,2,2$, $, \ldots,(2,2,2,2,1)$ form a clique which we choose as a line $S$. The remaining lines are the translates $S+c$ with $\underline{c} \in C$. Recently Cameron and van Lint [15] showed that the automorphism group of this geometry is the semidirect product of this translation group and $S_{6}$. 
The proof uses the following elegant description.

Let $\bar{C}$ be the ternary repetition code of length 6 . The sum of the coordinates of a vector in a coset of $\bar{C}$ is a constant which we call the type of the coset. Let $\mathbb{A}_{i}$ be the set of cosets of type $i$. We define a tripartite graph $\Gamma$ by joining the coset $\bar{C}+\underline{v}$ to the coset $\bar{C}+\underline{v}+\underline{w}$ for each vector $\underline{w}$ of weight 1 . Then any vector in $\mathbf{A}_{i}$ has six neighbours in $\mathbf{A}_{i+1}$ and six in $\mathbf{A}_{i+2}$. If we take any $\mathbf{A}_{i}$ as point set and $\mathbf{A}_{i+1}$ as line set and define incidence by adjacency, we find the $p g(6,6,2)$. In fact we now have three 'linked' copies of this geometry.

It is of interest to observe that $\Gamma$ is a subgraph of $\operatorname{srg}(243,22,1,2)$ constructed by Berlekamp et al. (Section 10D(i)).

\section{E. A New Infinite Sequence: $p g\left(2^{2 n-1}, 2^{2 n-1}+1,2^{2 n-2}\right)$}

Cohen [21] gave the first description of a $p g(8,9,4)$. It was (and still may be) sporadic. (Added in proof: Recently Tonchev [71a] showed the isomorphism of this $p g$ with the dual of the $p g$ described below.) Subsequently, Haemers and van Lint [37] gave a much simpler description of a $p g(9,8,4)$ as follows.

Take as vertices the words of weight 4 or 8 in $\left(I F_{2}\right)^{9}$ and join these by an edge if their distance is congruent to two (mod 4). Among the cliques of size 9 there are the sets corresponding to the rows of $(J-I)_{9}$,

$$
\left(\begin{array}{ccc}
0 & \underline{1} & \underline{1} \\
\underline{1}^{t} & (J-I)_{4} & 0 \\
\underline{1}^{t} & 0 & (J-1)_{4}
\end{array}\right) \text { and }\left(\begin{array}{ccc}
J_{3} & I_{3} & 0 \\
0 & J_{3} & I_{3} \\
I_{3} & 0 & J_{3}
\end{array}\right) \text { and permutations. }
$$

From these cliques $1+63+2.28=120$ are chosen as lines. In order to do this in a 'natural' way the 9 positions are identified with the points of $P G(1,8)$ and the action of $\operatorname{PSL}(2,8)$ is used. For details we refer to [37].

These examples led F. de Clerck, R.H. Dye and J.A. Thas to a third construction and subsequently the discovery of a new infinite sequence, namely the $p g\left(2^{2 n-1}, 2^{2 n-1}+1,2^{2 n-2}\right)$. The construction is as follows. Let $Q^{+}$be a hyperbolic quadric in $P G(4 n-1,2)$. The set of maximal totally isotropic subspaces of $Q^{+}$is divided into two disjoint families $D_{1}$ and $D_{2}$. If $H$ is a projective space of dimension $2 n-2$ on $Q^{+}$then $Q^{+}$contains two maximal totally isotropic subspaces through $H$, one of each family. Together they determine a $2 n$-space which contains a unique hyperplane $M(H)$ through $H$ and not on $Q^{+}$. We observe that $M(H) \cup H$ has $2^{2 n-1}$ points not on $Q^{+}$. Let $\Psi$ be a spread of $Q^{+}$consisting of elements of $D_{1}$. We define: 
$X:=\left\{\right.$ points not on $\left.Q^{+}\right\}, \mathrm{L}:=\{$ all spaces $M(H)$ where $H$ is a hyperplane in an element of $\Psi\}$.

We call the elements of $\mathbf{L}$ lines and take natural incidence. Then $(X, L)$ is a $p g\left(2^{2 n-1}, 2^{2 n-1}+1,2^{2 n-2}\right)$. For a proof we refer to [20].

The idea of using spreads or define srg's and $p g$ 's was generalized by Kantor (see Section 6E). He also proved that the partial geometry pg $(8,9,4)$ in the infinite sequence is isomorphic to the dual of the one constructed by Haemers and van Lint. They have $A_{9}$ as sutomorphism group!

F. A Possibly Infinite Sequence: $p g\left(3^{2 h+1}, 3^{2 h+1}+1,2 \cdot 3^{2 h}\right)$

In [71] J.A. Thas generalized the construction given in $11 \mathrm{E}$ above, using hyperbolic quadrics in $P G(4 h+3,3)$. Again the construction depends on the existence of spreads. Only for the cases $h=0$ and $h=1$ it is known that such spreads exist. The case $h=0$ leads to a trivial geometry but $h=1$ gives a new partial geometry $p g(27,28,18)$. At present this is a sporadic example.

\section{G. The sporadic geometry $p g(5,18,2)$}

A remarkable sporadic $p g$ was constructed by W. Haemers [35] using the Hoffman-Singleton graph. This graph contains 525 Petersen graphs as induced subgraphs. A special class of 105 of these is defined. Let the edges of the Hoffman-Singleton graph be the points of a geometry and define lines as 1-factors of the special Petrsen subgraphs. This produces $p g(5,18,2)$. More details were given in Section $10 \mathrm{~B}$.

\section{H. Some interesting open cases.}

In $11 \mathrm{~B}$ we mentioned the $p g(6,9,4)$ connected with the projective plane of order 10. It is expected that by the end of 1982 it will be known whether this $p g$ exists. (It is dead now.)

In the list of block designs in M. Hall's book [38] the first unknown design is number 35 : a $2-(46,6,1)$. If this design exists then it produces $p g(9,6,6)$ and $\operatorname{srg}(69,20,7,5)$ which are unknown.

The next unknown case is $p g(5,8,2)$ belonging to the unknown $\operatorname{srg}(75,32,10,16)$. Since this geometry has eight lines per point and the same number of lines as $p g(9,8,4)$ one might hope to find it by deleting a suitably chosen set of 60 points from $p g(9,8,4)$. In [37] it was shown that this idea does not work. 


\section{References}

[1] Belevitch, V., Theory of $2 n$-terminal networks with application to conference telephony, Elect. Commun. 27 (1950) 231-244.

[2] Benson, C.T. and N.E. Losey, On a graph of Hoffman and Singleton, J. Comb. Th. 11 (1971) 67.79.

[3] Berlekamp, E. R., J.H. van Lint and J.J. Seidel, A strongly regular graph derived from the perfect ternary Golay code, in: A survey of combinatorial theory, J.N. Srivastava e.a. (eds.), North-Holland 1973, pp. 25-30.

[4] Bose, R.C., Strongly regular graphs, partial geometries, and partially balanced designs, Pacific J. Math. 13 (1963) 389-419.

[5] Bose, R.C. and I.A. Dowling, A generalization of Moore graphs of diameter two, J. Comb. Th. (B) 11 (1971) 213-226.

[5a] Broue, M. and M. Enguehard, La geometrie de Graham Higman invariante par le groupe d'Higman-Sims, Exposé au Séminaire Claude Chevalley, Avril-Mai 1974.

[6] Brouwer, A.E., The uniqueness of the strongly regular graph on 77 points, Math. Centrum Report ZW 147/80, Amsterdam, 1980.

[7] Brouwer, A.E., Polarities of G. Higman's symmetric design and a strongly regular graph on 176 vertices, Math. Centrum Report ZW 158/81, Amsterdam, 1981.

[8] Brouwer, A.E. and A. Neumaier, Strongly regular graphs where $\mu=2$ and $\lambda$ is large, Math. Centrum Report 151/81, Amsterdam, 1981.

[8a] Brouwer, A.E., A. Schrijver and H. A. Wilbrink, personal communication.

[9] Bussemaker, F.C., W.H. Haemers, R. Mathon and H.A. Wilbrink, personal communication.

[9a] Cameron, P.J., Partial Quadrangles, Quart. J. Math. Oxford 26 (1975) 61-73.

[10] Cameron, P.J., Strongly regular graphs, in: Selected topics in graph theory, L.W. Beineke and R. J. Wilson (eds.), Academic Press 1978, pp. 337-360.

[10a] Cameron, P.J., personal communication.

[11] Cameron, P.J., J.-M. Goethals and J.J. Seidel, Strongly regular graphs having strongly regular subconstituents, J. Algebra 55 (1978) 257-280.

[12] Cameron, P.J., J.-M. Goethals and J.J. Seidel, The Krein condition, spherical designs, Norton algebras and permutation groups, Proc. KNAW A81 (=Indag. Math. 40 ) (1978) 196-206.

[13] Cameron, P.J., J.-M. Goethals, J.J. Seidel and E.E. Shult, Line graphs, root systems and elliptic geometry, J. Algebra 43 (1976) 305-327.

[14] Cameron, P.J. and J.H. van Lint, Graphs, Codes and Designs, London Math. Soc. Lecture Note Series 43. Cambridge 1980.

[15] Cameron, P.J. and J.H. van Lint, On the partial geometry pg(6,6,2), J. Comb. Th. (A) 32 (1982) 252-255.

[16] Chang, L.C., The uniqueness and nonuniqueness of triangular association schemes, Sci. Record 3 (1949) 604-613.

[17] Chang, L.C., Association schemes of partially balanced block designs with parameters $v=28, n_{1}=12, n_{2}=15$ and $p_{11}^{2}=4$, Sci. Record 4 (1950) $12-18$.

[18] Clatworthy, W.H., Partially balanced incomplete block designs with two associate classes and two treatments per block, J. Res. Nat. Bur. Standards 54 (1955) 177. 190. 
[19] Clerck, F. de, Partial Geometries, thesis, University of Ghent, 1978.

[20] Clerck, F. de, R.H. Dye and J.A. Thas, An infinite class of partial geometries associated with the hyperbolic quadric in $P G(4 n-1,2)$, Eur. J. Combinatorics 1 (1980) 323-326.

[21] Cohen, A.M., A new partial geometry with parameters $(s, t, \alpha)=(7,8,4)$, J. of Geometry 16 (1981) 181-186.

[22] Connor, W.S., The uniqueness of the triangular association scheme, Ann. Math. Stat. 29 (1958) 262-266.

[23] Cvetkovic, D.M., M. Doob and H. Sachs, Spectra of Graphs, Theory and Application, Academic Press, New York etc. 1980.

[24] Debroey, I., Serni partièle meetkunden, thesis, University of Ghent, 1978.

[25] Debroey, I. and J.A. Thas, On semi partial goemetries, J. Comb. Th. (A) 25 (1978) $242-250$.

[26] Delsarte, P., An algebraic approach to the association schemes of coding theory, Philips Res. Repts. Suppl. 10 (1973).

[27] Delsarte, P., J.-M. Goethals and J.J. Seidel, Bounds for systems of lines and Jacobi polynomials, Philips Res. Repts. 30 (1975) $91^{*}-105^{*}$.

[28] Dixmier, S. and F. Zara, Essai d'une methode d'etude de certains graphes liés aux groupes classiques, C. R. Acad. Sc. Paris 282 Série A, 259-262.

[29] Dixmier, S. and F. Zara, Etude d'un quadrangle généralisé autour deux de ses points non lies, preprint.

[29a] Faima, G. and G. Cecconi, A finite Buekenhout oval which is not projective, Simon Stevin 56 (1982) 121-127.

[30] Games, R., personal communication.

[31] Gewirtz, A., The uniqueness of $g(2,2,10,56)$, Trans. New York Acad. Sci. 31 (1969) 656-675.

[32] Gewirtz, A., Graphs with maximal even girth, Can. J. Math. 21 (1970) 915-934.

[33] Goethals, J.-M. and J.J. Seidel, Strongly regular graphs derived from combinatorial designs, Can. J. Math. 22 (1970) 597-614.

[34] Goethals, J.-M. and J.J. Seidel, The regular two-graph on 276 vertices, Discrete Math. 12 (1975) 143-158.

[35] Haemers, W.H., Eigenvalue techniques in design and graph theory, thesis (Eindhoven) $1979=$ Math. Centre Tract 121. Amsterdam 1980.

[36] Haemers, W.H. , A new partial geometry constructed from the Hoffman-Singleton graph, in: Finite Geometries and Designs, P.J. Cameron, J. W. P. Hirschfeld and D. R. Hughes (eds.), London Math. Soc. Lecture Note Series 49. Cambridge 1981, pp. 119-127.

[37] Haemers, W.H. and J.H. van Lint, A partial geometry $\operatorname{pg}(9,8,4)$, Annals of Discr. Math. 15 (1982) 205-212.

[38] Hall, M., Jr., Combinatorial Theory, Blaisdell 1967.

[39] Higman, D.G., Invariant relations, coherent configurations and related generalized polygons, in: Combinatorics, M. Hall, Jr. and J.H. van Lint (eds.), Math. Centre Tracts 57. Amsterdam 1974, pp. 27-43.

[40] Higman, G., On the simple group of D.G. Higman and C.C. Sims, Illinois J. Math. 13 (1969) 74-80. 
[41] Hill, R., Caps and codes, Discrete Math. 22 (1978) 111-137.

[42] Hoffman, A.J., On the uniqueness of the triangular association scheme, Ann. Math. Stat. 31 (1960) 492-497.

[43] Hoffman, A.J. and R. R. Singleton, On Moore graphs with diameter 2 and 3 , IBM J. Res. Develop. 4 (1960) 497.504.

[44] Hollman, H.D.L., Association schemes, Master's thesis, Eindhoven University of Technology, 1982.

[45] Hubaut, X.L., Strongly regular graphs, Discrete Math. 13 (1975) 357-381.

[46] Kageyama, S., G.M. Saha and A.D. Das, Reduction of the number of association classes of hypercubic association schemes, Ann. Inst. Stat. Math. 30 (1978) 115 123.

[47] Kantor, W.M., Strongly regular graphs defined by spreads, Isr. J. Math. 41 (1982) 298-312.

[48] Kantor, W.M. and R. A. Liebler, The rank three representations of the finite classical groups, Trans. Amer. Math. Soc. 271 (1982) 1-71.

[49] Koornwinder, T.H., A note on the absolute bound for systems of lines, Proc. KNAW A 79 (= Indag. Math. 38 ) (1976) 152-153.

[50] Kreïn, M. G., Hermitian-positive kernels II, Amer. Math. Soc. Transl. Ser. 234 (1963) pp. 109-164 (in particular p. 139).

[50a] Lam, C.W.H., L. Thiel, S. Swiercz and J. McKay, The nonexistence of ovals in a projective plane of order 10, Discr. Math. 45 (1983) 319-321.

[51] Lint, J.H. van and J.J. Seidel, Equilateral point sets in elliptic geometry, Proc. KNAW A 69 (=Indag. Math. 28 ) (1969) 335-348.

[52] Lint, J.H. van and A. Schrijver, Construction of strongly regular graphs, twoweight codes and partial geometries by finite fields, Combinatorica 1 (1981) 63-73.

[53] Mathon, R., Three-class association schemes, in: Proc. Conf. on Algebraic Aspects of Combinatorics, Toronto 1975, pp. 123-155.

[54] Mathon, R., Symmetric conference matrices of order $p q^{2}+1$, Can. J. Math 30 (1978) 321-331.

[55] Mathon, $\mathrm{R}$., The partial geometries $\mathrm{pg}(5,7,3)$, Congressus Numerantium 31 (1981) 129-139.

[55a] Mathon, R., personal communication.

[55b] Mathon, R. and A. Rosa, A census of 1-factorizations of $K_{9}^{3}$ with automorphism groups of order $>4$, in preparation.

[56] Mesner, D.M., A new family of partially balanced incomplete block designs with some Latin square properties, Ann. Math. Stat. 38 (1967) 571-581.

[57] Neumaier, A., Strongly regular graphs with smallest eigenvalue $-m$, Archiv der Mathematik 33 (1979) 392-400.

[58] Neumaier, A., New inequalities for the parameters of an association scheme, Combinatorics and Graph Theory, Springer Lecture Notes 885 p. 365-367.

[59] Robertson, N., personal communication.

[60] Scott, L.I., A condition on Higman's parameters, Notices Amer. Math. Soc. (1973) 701-20-45 (page A-97).

[61] Seidel, J.J., Strongly regular graphs with $(-1,1,0)$ adjacency matrix having 
eigenvalue 3, Lin. Algebra and Appl. 1 (1968) 281-298.

[62] Seidel, J.J., A survey of two-graphs, in: Proc. Intern. Colloq. Theorie Combinatorie (Roma 1973), tomo I, Accad. Naz. Lincei, 1976, pp. 481-511.

[63] Seidel, J.J., Strongly regular graphs, in: Surveys in Combinatorics, Proc. 7th Brit. Comb. Conf., B. Bollobás (ed.), London Math. Soc. Lecture Note Series 38. Cambridge 1979, pp. 157-180.

[64] Seidel J.J. and D.E. Taylor, Two-graphs, a second survey, in: Proc. Intern. Colloq. Algebaic Methods in Graphs Theory, Szeged 1978, Coll. Math. Soc. Bolyai 25. pp. 689-711.

[65] Shrikhande, S.S., The uniqueness of the $L_{2}$ association scheme, Ann. Math. Stat. 30 (1959) 781-798.

[66] Sims, C.C., On the isomorphism of two groups of order 44, 352, 000, in: Theory of Finite Groups, Brauer and Sah (eds.), Benjamin 1969.

[67] Smith, M.S., On the isomorphism of two simple groups of order 44, 352, 000, J. Algebra 41 (1976) 172-174.

[68] Taylor, D.E., Graphs and block designs associated with the three-dimensional unitary groups, in: Combinatorial Mathermatics, Proc. 2nd Austr. Conf., D. A. Holton (ed.), Springer Lecture Notes 403. Berlin etc. 1974, pp. 128-131.

[69] Taylor, D.E., Regular 2-graphs, Proc. London Math. Soc. 35 (1977) 257-274.

[70] Thas, J.A., Combinatorics of partial geometries and generalized quadrangles, in: Higher Combinatorics, M. Aigner (ed.), Reidel, Dordrecht 1977, pp. 183-199.

[71] Thas, J.A., Some results on quadrics and a new class of partial geometries, Simon Stevin 55 (1981) 129-139.

[71a] Tonchev, V.D., The isomorphism of Cohen, Haemers-van Lint and De ClerckDye-Thas partial geometries, submitted to Discr. Math.

[72] Wallis, W.D., A.P. Street and J. Seberry Wallis, Combinatorics: room squares, sum-free sets, Hadamard matrices, Springer Lecture Notes 292. Berlin etc. 1972.

[73] Wilbrink, H.A. and A.E. Brouwer, A $(57,14,1)$ strongly regular graphs does not exist, KNAW A 86 (=Indag. Math. 45) (1983) 117-121.

[74] Wilbrink, H.A., personal communication.

[75] Zara, F., personal communication. 